\title{
PHYSIOTHÉRAPIE SUISSE : VERS UN ACCÈS DIRECT ?
}

\author{
Valentin SARRASIN \\ Etudiant HES - Filière Physiothérapie
}

Philippe OGAY

Etudiant HES - Filière Physiothérapie

Directeurs de travail de Bachelor :

Seraina OBRIST \& Etienne DAYER

TRAVAIL DE BACHELOR POUR L'OBTENTION DU DIPLÔME

BACHELOR OF SCIENCE HES-SO EN PHYSIOTHERAPIE

JUILLET 2013 


\section{Résumé}

\section{Introduction}

L'accès direct en physiothérapie permet à un patient de se faire traiter par un physiothérapeute sans avoir consulté de médecin au préalable. Cette modalité de parcours de soin n'existe pas en Suisse mais est instaurée dans plusieurs pays d'Europe, aux USA, au Canada et en Australie.

\section{Objectifs}

Le but de cette revue narrative est de déterminer s'il existe une différence entre le profil d'une personne consultant son physiothérapeute en accès direct et celui d'un patient qui sollicite son médecin au préalable. Puis, selon nos résultats, nous essaierons de définir un profil type de patient consultant son physiothérapeute en accès direct.

\section{Méthode}

Les moteurs de recherche Pubmed, Web of Knowledge, Science Direct, Psychinfo, Cochrane, PEDro, Medline, Francis et CINAHL ont été consultés, donnant un total de 482 articles. Après une sélection comportant trois tris et une évaluation de la qualité avec une version modifiée de l'échelle «Critical Review Form » de Law, cinq articles ont été retenus.

\section{Résultats}

Les résultats montrent une différence de profil entre les deux groupes, caractérisée par les critères âge, diagnostic, durée des symptômes, arrêt du traitement et réussite des objectifs.

\section{Conclusion}

Le profil type d'une personne consultant son physiothérapeute en accès direct se démarque par une donnée personnelle - l'âge - et une donnée clinique - le diagnostic. L'accès direct offre de nombreux avantages tels que des soins rapides et de qualité, une diminution des coûts de la santé et une amélioration de la collaboration entre les physiothérapeutes et les autres professionnels de la santé.

Mots-clés : Accès direct, physiothérapie, profil 


\section{Zusammenfassung}

\section{Einführung}

Der Direktzugang zur Physiotherapie ermöglicht einem Patienten ohne ärztliche Überweisung und vorgegebene Diagnose behandelt zu werden. Dieser Direktzugang existiert nicht in der Schweiz, ist aber schon vorhanden in mehreren Ländern Europas, den USA, Kanada und Australien.

\section{Ziele}

Das Ziel dieser Review ist festzulegen, ob es einen Unterschied gibt zwischen Direktzugang Patienten und ärztlich überwiesene Patienten. Gemäss erhaltenen Resultaten wird dann versucht, ein typisches Patientenprofil im Direktzugang festzulegen.

\section{Methode}

Die Datenbanken Pubmed, Web of Knowledge, Science Direct, Psychinfo, Cochrane, PEDro, Medline, Francis und CINAHL wurden konsultiert, was ein Total von 482 Studien ergibt. Nach dreimaligem Sortieren und einer Auswahl nach Qualitätsbewertung anhand einer geänderten Version der «Critical Review Form » Skala von Law, wurden schlussendlich fünf Studien zurückbehalten.

\section{Resultaten}

Die Resultate zeigen einen Profilunterschied zwischen beiden Gruppen. Er betrifft das Alter, die Diagnose, die Zeitdauer der Symptome, das Absetzen der Behandlung und das Erreichen der Zielsetzung.

\section{Schlussfolgerung}

Das typische Profil eines Direktzugang Patienten äussert sich in einem persönlichen Faktor - dem Alter - und einem klinischen Faktor - der Diagnose. Der Direktzugang zur Physiotherapie bietet mehrere Vorteile: rasche und optimale Behandlung, verminderte Gesundheitskosten und eine verbesserte Zusammenarbeit zwischen Physiotherapeuten und anderen Gesundheitsfachleuten.

Schlüsselwörter: Direktzugang, Physiotherapie, Profil 


\section{Summary}

\section{Introduction}

Direct access to physical therapy allows a patient to be treated by a physiotherapist without consulting a doctor first. This form of care protocol does not exist in Switzerland, but has been introduced in several European countries as well as in the United States, Canada and Australia.

\section{Objectives}

The aim of this study is to determine if there is a difference between the profile of a person who consults a physiotherapist directly and a patient who goes to see his/her doctor first. Then, according to our results, we will try to define a typical profile of a patient who consults a physiotherapist in direct access.

\section{Method}

Search engines Pubmed, Web of Knowledge, Science Direct, Psychinfo, Cochrane, PEDro, Medline, CINAHL and Francis were consulted, giving a total of 482 articles. After a selection in three stages and a quality assessment with a modified version of the scale "Critical Review Form" of Law, five articles have been selected.

\section{Results}

The results show a significant difference between the two groups' profiles, characterized by age, diagnosis, duration of symptoms, discontinuation of the treatment and achievement of the objectives.

\section{Conclusion}

The profile type of a person from the "direct access" group stands out by personal information - age - and clinical data - diagnosis. Direct access in physiotherapy has advantages such as quick and quality care, reduced health care costs and better collaboration between physiotherapists and other health care professionals.

Keywords: Direct access, physical therapy, profile 


\section{Remerciements}

A Seraina Obrist et Etienne Dayer, nos responsables et enseignants à la HES-SO Valais, pour leur soutien et leur conseil durant cette longue année.

A Lara Allet et Elisabeth Bürge, toutes deux enseignantes à la HEdS, pour leur aide précieuse en matière d'accès direct et pour leur disponibilité.

A Philippe Germanier, président de Physiovalais, pour avoir lu notre travail et pour ses précieux commentaires.

A Richard Ogay, pour avoir pris le temps de lire notre travail et pour ses précieuses corrections.

A Eléonore Ribordy, pour son aide dans les traductions français-anglais et pour les corrections apportées à notre travail.

A Gabrielle Hager-Mittaz, enseignante à la HES-SO Valais, pour son aide dans les échelles de qualité et la méthodologie.

A Isle Swinkels, chercheuse à l'institut des Services de Santé aux Pays-Bas, pour ses références d'articles concernant l'accès direct.

A Valerie Webster, professeur à l'université de Glasgow, pour ses références d'articles concernant l'accès direct.

Et à toutes les autres personnes qui nous ont, d'une manière ou d'une autre, aidés et soutenus dans ce travail. 


\section{Lexique}

\begin{tabular}{|c|c|c|}
\hline Accès direct & $=$ & $\mathrm{AD}$ \\
\hline Physiothérapeute & $=$ & PT \\
\hline Médecin généraliste & $=$ & $\mathrm{MG}$ \\
\hline World Confederation for Physical Therapy & $=$ & WCPT \\
\hline Traitement & $=$ & TTT \\
\hline Musculo-squelettique & $=$ & MSQ \\
\hline Evidence Based Medicine & $=$ & EBM \\
\hline Radiographie & $=$ & $\mathrm{Rx}$ \\
\hline Ultrason & $=$ & US \\
\hline
\end{tabular}




\section{Table des matières}

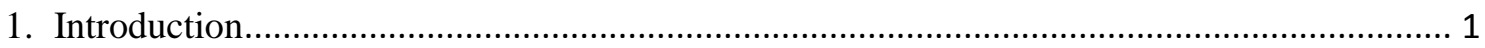

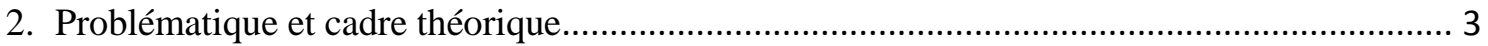

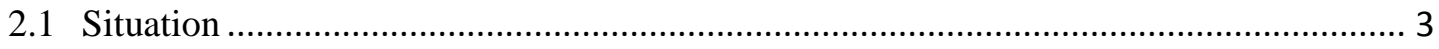

2.2 Définition de l'accès direct en physiothérapie ............................................................... 4

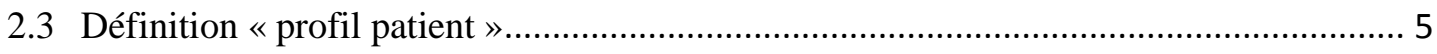

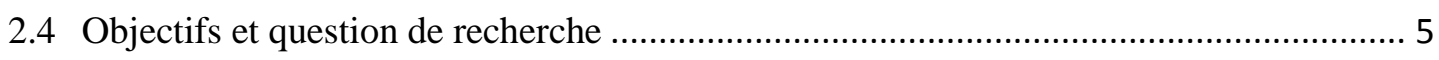

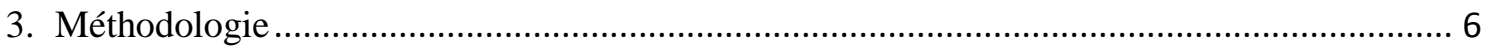

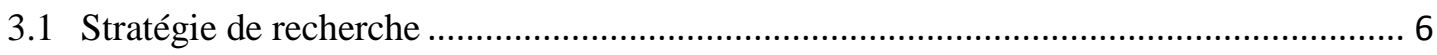

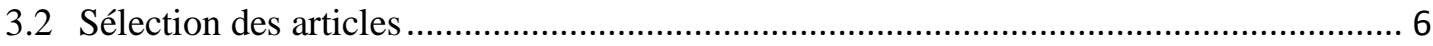

3.3 Evaluation de la qualité ………................................................................................... 7

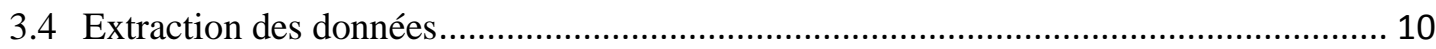

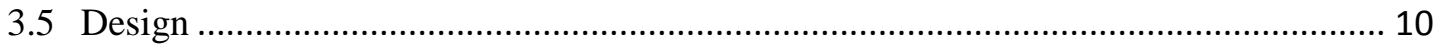

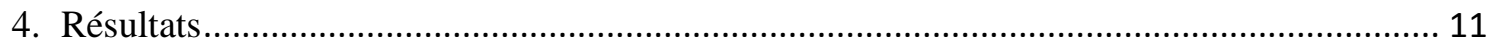

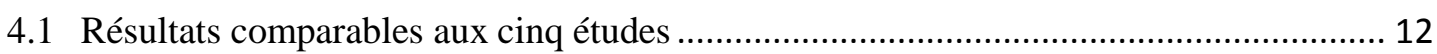

4.2 Résultats comparables à quatre études ........................................................................ 14

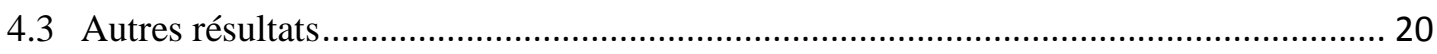

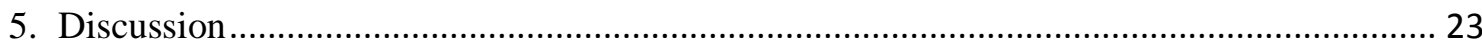

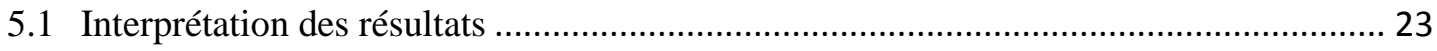

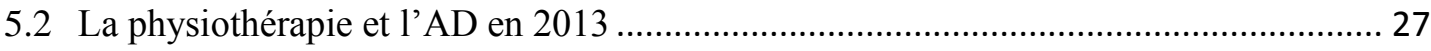

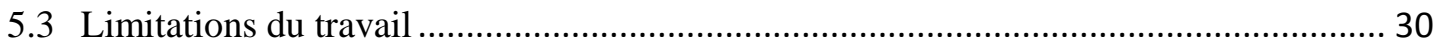

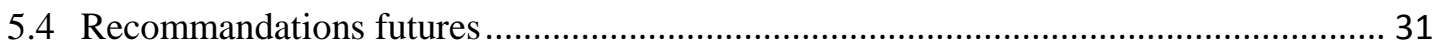

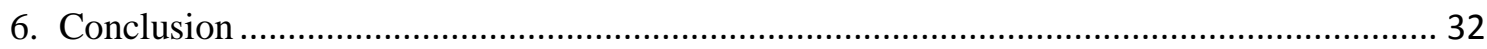

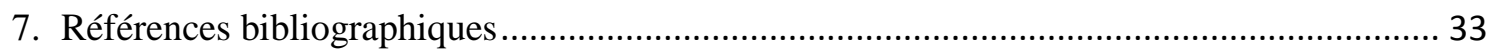

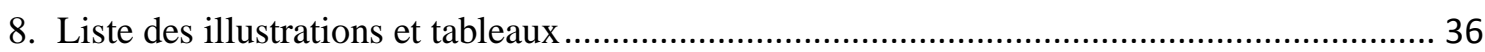

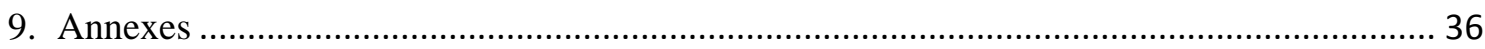




\section{Introduction}

En 2011, la Suisse comptait environ 11'000 physiothérapeutes, dont 5'564 dans le secteur de soins ambulatoires (Huber, 2011, p. 93). Dans la même année, la Fédération des médecins suisses a enregistré un total de 30'849 médecins exerçant en Suisse dans les secteurs hospitaliers, ambulatoires ou autres. La population résidant en suisse en 2011 était de 7’954'700 personnes (Office fédéral de la statistique $[\mathrm{OFS}], 2013)$. Ces chiffres ont évolué de manière proportionnelle et démontrent que le nombre de physiothérapeutes (Huber, 2011, p. 93) et de médecins (Fédération des médecins suisses [FMH], 2013) par habitant est adéquat.

Mais ces chiffres masquent une réalité :

Tout d'abord, nous manquons de médecins généralistes. Principal guide du parcours de soin dans notre système de santé, ils étaient au nombre de 5'800 en 2011, soit environ 19\% de la totalité des médecins (FMH, 2013). Ce pourcentage est très en retrait des 50\% qu'affichaient en 2009 des pays comme la France, le Portugal, l'Australie et le Canada ou les 30\% de l'Autriche, du Royaume-Uni et du Luxembourg (Organisation de Coopération et de Développement Économiques [OCDE], 2013). La nouvelle génération de médecins préfère se spécialiser. PierreFrançois Unger, conseiller d'Etat genevois, s'est d'ailleurs exprimé à ce sujet en disant : «Nous manquons de généralistes alors que la santé publique est envahie de spécialistes dont elle n'a pas besoin »(L'HEBDO, 2012). En Suisse et ailleurs, certaines régions rurales sont de véritables déserts lorsqu'il s'agit de trouver des médecins généralistes.

Ensuite, Le deuxième problème est l'âge moyen de nos médecins. Les chiffres montrent que la moyenne d'âge est de quarante-cinq ans pour les femmes et de cinquante et un ans pour les hommes. Celui des médecins dans le secteur ambulatoire - comme les médecins généralistes - est de cinquante-trois ans (FMH, 2013). D’ici une vingtaine d'années, la plupart des médecins généralistes prendront leur retraite; qui occupera les places vacantes dans les cabinets oeuvrant au sein des villages? Seematter-Bagnoud et al. concluent d'ailleurs dans une de leurs recherches (2010) que d'ici 2030, 30\% des consultations médicales ne pourront plus être assurées et ceci à cause du manque de médecin généraliste. Une solution serait de redistribuer les rôles de la santé. 
Dans certains pays, les patients peuvent accéder directement à leur physiothérapeute pour recevoir les premiers soins. Sans qu'un médecin ait été au préalable consulté et sans diagnostic préétabli, les physiothérapeutes offrent des prestations remboursées par le biais du système de santé public ou privé. Dans les pays où cet accès direct en physiothérapie est en vigueur, des analyses ont démontré que ce système amenait un certain nombre d'avantages (Scheermesser, 2011, pp. 220-1).

Depuis plusieurs années, l'accès direct suscite l'intérêt des physiothérapeutes suisses. D'ailleurs, en 1998, le thème d'un congrès à Lausanne était : « Accès direct à la physiothérapie : Utopie ou réalité de demain ?» (Larequi, 1999). 


\section{Problématique et cadre théorique}

\subsection{Situation}

Les physiothérapeutes suisses doivent faire face à des défis importants et doivent prendre en considération de grands changements dans leur pratique quotidienne. L'augmentation rapide des coûts de la santé ces dernières années (plus de soixante milliards de dépenses en 2010, à savoir 10\% du PIB suisse) (OFS, 2013), la pyramide des âges inversée qui évolue vers un vieillissement massif de la population ainsi que l'augmentation des maladies chroniques en sont quelques exemples. Sans parler de la tourmente juridique qui oppose santésuisse aux physiothérapeutes, au sujet de la valeur - inchangée depuis 1998 - du point tarifaire, alors que les coûts de la vie ont augmenté de 11,3\% entre 1998 et 2008 (Oggier, 2011, p. 89). C'est d'ailleurs l'un des grands thèmes actuels de la campagne politique de l'Association Suisse des Physiothérapeutes Indépendants, Physioswiss. Cette dernière a calculé que le revenu net des physiothérapeutes indépendants aurait chuté de $27 \%$ entre 1998 et 2008, en prenant en considération l'augmentation générale des revenus du système de santé et les coûts de la vie (Paillex, 2011, p. 89).

De plus, on s'attend à ce que d'ici 2030, 30\% des consultations médicales ne puissent plus être assurées notamment à cause du manque grandissant de médecins généralistes et de personnel soignant qualifié. (Seematter-Bagnoud, 2010). A long terme, cela conduirait à moins de possibilités de consultations pour les patients. C'est pourquoi l'idée tend vers une redistribution des rôles des divers acteurs de la santé, afin de maîtriser au mieux les défis futurs (Scheermesser et al., 2011, p. 30).

Les expériences faites dans les pays où l'accès direct en physiothérapie est en vigueur ont permis de mettre en évidence plusieurs avantages. Il serait en effet question: de gestion des coûts améliorée, de soins rapides et adéquats, de satisfaction des patients augmentée, de diminution des rendez-vous médicaux et des clichés radiologiques. (Scheermesser, 2012, p. 221). Physioswiss a mandaté un groupe d'étude sur l'accès direct, afin de pouvoir en objectiver au mieux les avantages, les inconvénients et les risques (Physioswiss, 2011). Un projet devrait voir le jour prochainement et s'étendre sur une année et demie dans deux cantons pilotes. 
Pour notre travail, nous voulions traiter un thème d'actualité qui touche de près les physiothérapeutes et qui permette une projection dans le futur de la profession. Nous en sommes donc venus sur l'accès direct en physiothérapie. Avant de nous interroger sur les coûts de la santé, la politique, le domaine assécurologique ou un quelconque autre aspect, nous nous sommes demandé quelles seraient les personnes intéressées par l'accès direct et lesquelles auraient tendance à consulter leur physiothérapeute en première instance plutôt que leur médecin. Le but est de dégager, si possible, un profil type. Ce qui nous amène à définir plus en détail ce qu'est l'accès direct et ce que nous pouvons comprendre par un profil.

\subsection{Définition de l'accès direct en physiothérapie}

Selon la WCPT, « the patient/client directly asks the physical therapist to provide services (the patient refers themselves) and the physical therapist freely decides his conduct and takes full responsibility for it »(2010). Pour une définition en français : «Par accès direct, il faut comprendre la possibilité pour les patients d'obtenir des traitements de physiothérapie directement, sans consultation médicale ni prescription préalable » (Scheermesser et al., 2012, p. $30)$.

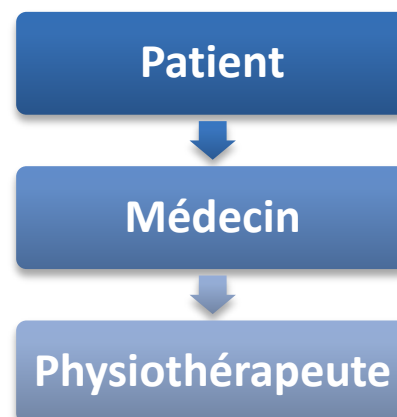

Figure 1 Parcours de soin en Suisse

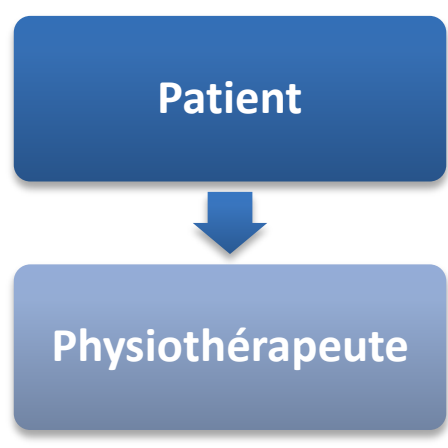

Figure 2 Parcours de soin avec AD

Les Australiens sont les précurseurs de l'accès direct en physiothérapie depuis 1976. Quarante-cinq Etats des USA l'ont introduit depuis 1985 et le Canada en 1999. En Europe, des projets pilotes ont été réalisés dans les années nonante. Ces derniers ont révélé des conclusions convaincantes, à tel point que la Suède a introduit l'accès direct dans ses provinces depuis 1996 (quatorze provinces sur vingt en 2004), puis la Norvège en 2005 et les Pays-Bas en 2006. La Grande-Bretagne dispose d'un projet pilote depuis 2003 en Ecosse et depuis 
2005 en Angleterre. L'Italie mène un projet pilote en Sardaigne depuis 2009 (Scheermesser, 2011, p. 220).

En Suisse, le patient doit être en possession d'une prescription médicale s'il tient à ce que ces séances de physiothérapie soient remboursées par son assurance maladie de base. Seules quelques assurances complémentaires remboursent des prestations à but préventif, comme le massage, le drainage lymphatique et l'acupuncture.

\subsection{Définition «profil patient »}

Le profil d'une personne est l'ensemble des caractéristiques qui la définissent fondamentalement (Larousse, 2013). Dans la littérature, le «profil patient » est composé de plusieurs catégories, elles-même composées de plusieurs éléments, qui déterminent le patient dans son intégralité :

1) Données personnelles : sexe, âge, niveau d'éducation, emploi, etc.

2) Données démographiques : habitation dans une zone urbaine, semiurbaine ou rurale.

3) Données cliniques : diagnostic, degré de sévérité, durée des symptômes, VAS, but de la thérapie atteint, etc.

Dans notre travail, nous utiliserons en priorité les données objectives et fréquemment mentionnées dans les études sélectionnées pour répondre aux mieux à nos objectifs.

2.4 Objectifs et question de recherche

Le but de notre travail est de déterminer s'il existe une différence entre le profil d'une personne consultant son physiothérapeute en accès direct et celui d'un patient qui sollicite son médecin au préalable. Puis, selon nos résultats, nous essaierons de définir un profil type de patient consultant son physiothérapeute en accès direct.

Quels éléments différencient le profil d'un patient allant chez son physiothérapeute en accès direct d'une personne consultant son médecin au préalable? 


\section{Méthodologie}

3.1 Stratégie de recherche

Pour répondre à notre question de recherche et en respectant la structure PICO (Population-Intervention-Comparaison-Outcomes) [annexe 1] définie, les études devaient comporter : une description des patients utilisant l'accès direct en physiothérapie, une définition d'un profil type et une comparaison entre ce profil et celui d'un patient passé au préalable chez son médecin.

Plusieurs bases de données ont été consultées, à savoir Pubmed, Web of Knowledge, Science Direct, Psychinfo, Cochrane, PEDro, Medline, Francis et CINAHL, en plus des références proposées sur le sites de la WCPT en rapport avec l'AD. L'équation de recherche [annexe 2] a été formulée par les termes anglophones désignant la physiothérapie, à savoir «physiotherapy » et « physical therapy », et les termes anglophones désignant l'accès direct selon la WCPT : «direct access », «self referral» et «first contact». Le terme Booléen « OR » a été utilisé pour combiner les synonymes et le terme « AND » pour les associer.

Nous avons décidé d'exclure les études qualitatives, car elles ne peuvent pas donner des outcomes quantifiés sous forme de chiffres et utilisables pour notre travail. Nous avons aussi rejeté les études randomisées-contrôlées (RCT), car les patients sont sélectionnés et répartis de manière homogène. Ce design ne correspond pas à notre travail qui tend à vouloir définir un profil type de patient allant chez son physiothérapeute en accès direct, sans contrôle ni sélection préalables. Nos avons aussi exclu les articles s'exprimant dans une autre langue que l'allemand, le français et l'anglais. Nous avons donc inclus des études de tous designs confondus (autre que RCT et qualitatif), qui traitent de l'accès direct en physiothérapie et décrivent un profil des patients concernés.

Nos recherches ont abouti à un total de 482 articles.

\subsection{Sélection des articles}

Notre premier tri a consisté à supprimer les doublons entre les bases de données et à garder les études rédigées en français, en anglais et en allemand. Nous sommes passés de 482 articles à 359. 
Notre deuxième tri a été réalisé en lisant les résumés des différentes études. Nous avons contrôlé si les articles avaient bien pour thème l'accès direct et la physiothérapie. Il nous est resté alors un total de quarante-sept articles.

Notre troisième et dernier tri a consisté à cibler les articles susceptibles de répondre à nos objectifs et notre question de recherche. Après relecture, nous avons gardé cinq articles.

\subsection{Evaluation de la qualité}

Nous avons choisi de travailler avec l'échelle de qualité «Critical Review Form - Quantitative Studies » de Law et al [annexe 3]. Cette échelle nous permet d'évaluer des études de différents designs, car dans notre travail, aucun design - hormis les RCT et les études qualitatives - n'était un critère d'exclusion. De plus, cette échelle est simple d'utilisation et les items sont clairs et décrits de manière précise par le guide qui l'accompagne.

Une fois l'échelle de Law choisie, nous avons réfléchi à la manière de la quantifier, afin que nous puissions noter facilement nos études. Une cotation permettrait d'objectiver la qualité de nos études et ainsi de les sélectionner ou non dans notre revue.

\section{Description de l'échelle «Critical Review Form - Quantitative Studies »}

Cette échelle est composée de huit items : study purpose, literature, design, sample, outcomes, intervention, results et conclusions. Chacun de ces items est défini sous forme de questions auxquelles on répond par: yes, no, not addressed ou not applicable (N/A). Un espace libre pour la justification des réponses se trouve à côté de ces questions.

Nous avons modifié la version de base de l'échelle [annexe 4] en la quantifiant. Ainsi, nous avons attribué 1 point pour yes, -1 point pour no, 0 point pour not addressed et N/A. Etant donné que les RCT faisaient partie de nos critères d'exclusion, nous n'avons pas accordé de point à ce design. Les autres designs, selon leur importance dans la pyramide de l'évidence, ont été notés de la manière suivante : 3 points pour les cohort studies, 2 points pour les casecontrols, 1 point pour les cross-sectionals, les single case designs et les before and after, et 0 point pour les case studies. Les valeurs maximales ont été additionnées, donnant un total de 17 points. Etant donné que nos études ne 
répondaient pas à certains items, comme la contamination ou la cointervention dans l'item intervention, la valeur maximale était plutôt de 15 points. Nous avons donc adapté notre barème de sélection et avons décidé d'un seuil minimal de 10 points. Les études ne répondant pas à ce critère minimal ont été exclues définitivement.

Nous avons évalué nos cinq articles avec cette échelle. Ayant défini la qualité voulue entre 10 et 17 points, nous avons été satisfaits de voir que chaque étude atteignait le score de sélection. Au final, nous avons donc eu cinq articles sélectionnés pour notre travail.

\begin{tabular}{|c|c|c|c|}
\hline Auteur & Titre & Année & Note \\
\hline $\begin{array}{l}\text { Holdsworth } \\
\text { et al. }\end{array}$ & $\begin{array}{l}\text { Direct access to physiotherapy in primary } \\
\text { care : now ?-and into the future? }\end{array}$ & 2004 & $12 / 17$ \\
\hline $\begin{array}{l}\text { Holdsworth } \\
\text { et al. }\end{array}$ & $\begin{array}{l}\text { Self-referral to physiotherapy: deprivation } \\
\text { and geographical setting. Is there a } \\
\text { relationship ? Results of a national trial. }\end{array}$ & $2005(1)$ & $12 / 17$ \\
\hline $\begin{array}{l}\text { Holdsworth } \\
\text { et al. }\end{array}$ & $\begin{array}{l}\text { Are patients who refer themselves to } \\
\text { physiotherapy different from those referred } \\
\text { by GPs? Results of a national trial. }\end{array}$ & $2005(2)$ & $12 / 17$ \\
\hline $\begin{array}{l}\text { Leemrisje } \\
\text { et al. }\end{array}$ & $\begin{array}{l}\text { Direct Access to Physical Therapy in the } \\
\text { Netherlands : Results From the First Year } \\
\text { in Community-Based Physical Therapy }\end{array}$ & 2008 & $13 / 17$ \\
\hline $\begin{array}{l}\text { Pendergast } \\
\text { et al. }\end{array}$ & $\begin{array}{l}\text { A Comparison of Health Care Use for } \\
\text { Physician-Referred and Self-Referred } \\
\text { Episodes of Outpatient Physical Therapy }\end{array}$ & 2011 & $11 / 17$ \\
\hline
\end{tabular}

Figure 3 Etudes sélectionnées

Pour simplifier la lecture, seul l'auteur principal de chaque étude est cité dans le tableau. Nous procéderons de la même manière dans les résultats et la discussion. Nous précisons que les deux études de l'auteur Holdsworth parues en 2005 ont le même échantillon de base et sont complémentaires, l'une étant la suite de l'autre. Nous parlerons de deux études, car leurs outcomes sont différents, mais n'utiliserons qu'une seule fois l'échantillon pour comptabiliser nos résultats. Etant donné que l'année de parution est la même pour les deux, nous leur avons rajouté un numéro ((1), (2)) afin de les différencier. 


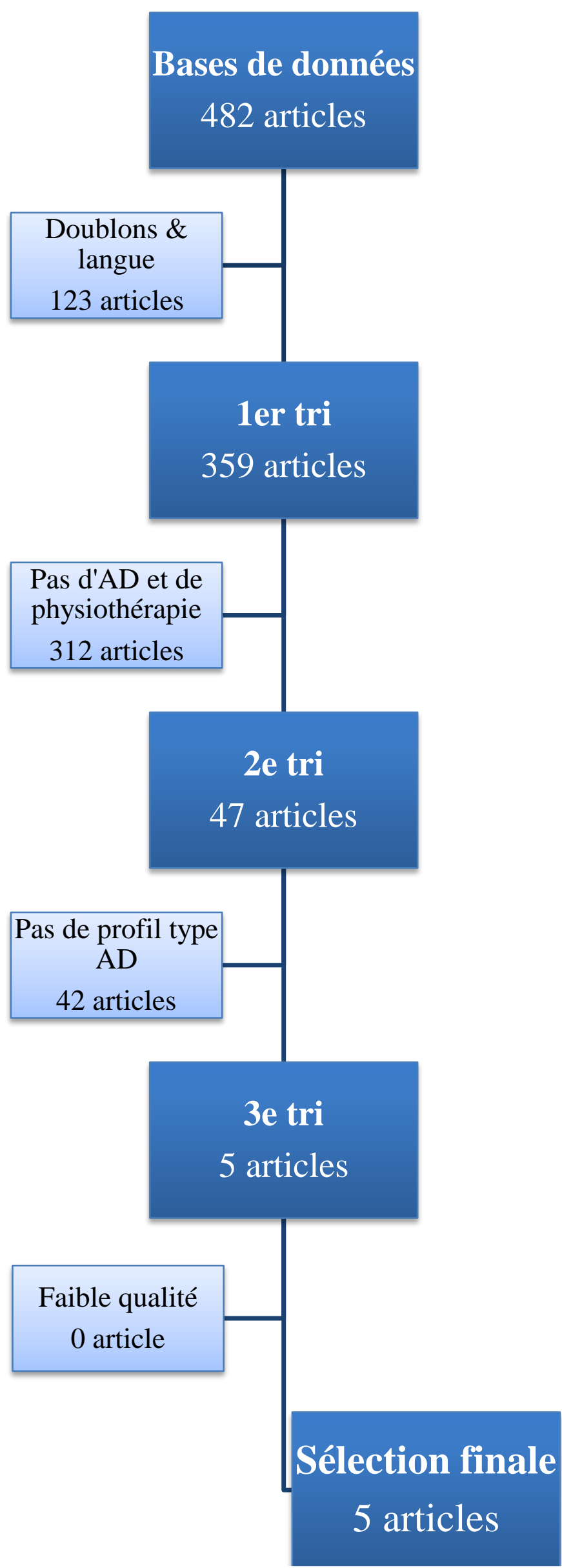

Figure 4 Organigramme de la sélection des articles 
3.4 Extraction des données

Nos cinq articles ayant été sélectionnés, nous sommes passés à l'extraction des données. Connaissant les études de manière globale, nous nous sommes concentrés sur les détails qui nous intéressaient. Grâce à notre grille d'extraction [annexe 5], nous avons pu isoler les outcomes relatifs au profil des patients utilisant l'accès direct et des patients consultant leur médecin en les regroupant sous quatre catégories : données personnelles, données cliniques, données démographiques et autres données lorsque des informations spécifiques à l'étude et intéressantes étaient proposées.

L'étude de Holdsworth de 2005 (2) propose une catégorie «GP-suggested referral group ». Ce groupe comporte les patients dont l'auteur ne connaissait pas vraiment leur mode de référence ( $\mathrm{AD}$ ou $\mathrm{MG}$ ). Nous avons donc décidé d'exclure ce groupe, car ce résultat n'est ni compatible avec le groupe AD ni MG. En plus, il ne représente que 18\% du total de l'échantillon.

Après avoir collecté toutes ces données, nous les avons regroupées dans un seul et même tableau, semblable à la grille d'extraction.

\subsection{Design}

Notre travail est une revue narrative de la littérature. 


\section{Résultats}

Nous avons regroupé les résultats des différentes études dans un tableau pour avoir une vue d'ensemble et ainsi mettre en avant les critères comparables entre le plus grand nombre d'études.

\begin{tabular}{|lccccc|}
\hline & $\begin{array}{c}\text { Holdsworth } \\
2004\end{array}$ & $\begin{array}{c}\text { Holdsworth } \\
2005(1)\end{array}$ & $\begin{array}{c}\text { Holdsworth } \\
2005(2)\end{array}$ & $\begin{array}{c}\text { Leemrisje } \\
2008\end{array}$ & $\begin{array}{c}\text { Pendergast } \\
2011\end{array}$ \\
\hline Sexe & $\mathrm{X}$ & $\mathrm{X}$ & $\mathrm{X}$ & $\mathrm{X}$ & $\mathrm{X}$ \\
\hline $\begin{array}{l}\text { MSQ ou } \\
\text { autres }\end{array}$ & $\mathrm{X}$ & $\mathrm{X}$ & $\mathrm{X}$ & $\mathrm{X}$ & $\mathrm{X}$ \\
\hline Âge & $\mathrm{X}$ & $\mathrm{X}$ & $\mathrm{X}$ & $\mathrm{X}$ & $\mathrm{X}$ \\
\hline $\begin{array}{l}\text { Diagnostics } \\
\text { MSQ }\end{array}$ & $\mathrm{X}$ & $\mathrm{X}$ & $\mathrm{X}$ & $\mathrm{X}$ & \\
\hline $\begin{array}{l}\text { Durée des } \\
\text { symptômes }\end{array}$ & $\mathrm{X}$ & $\mathrm{X}$ & $\mathrm{X}$ & $\mathrm{X}$ & \\
\hline Arrêt du TTT & $\mathrm{X}$ & $\mathrm{X}$ & $\mathrm{X}$ & $\mathrm{X}$ & \\
\hline $\begin{array}{l}\text { Réussite des } \\
\text { objectifs }\end{array}$ & $\mathrm{X}$ & $\mathrm{X}$ & $\mathrm{X}$ & $\mathrm{X}$ & \\
\hline $\begin{array}{l}\text { Zone } \\
\text { géographique } \\
\text { du cabinet }\end{array}$ & & $\mathrm{X}$ & & $\mathrm{X}$ & \\
\hline $\begin{array}{l}\text { Niveau de } \\
\text { formation du } \\
\text { patient }\end{array}$ & & $\mathrm{X}$ & & $\mathrm{X}$ & \\
\hline $\begin{array}{l}\text { Statut } \\
\text { professionnel }\end{array}$ & $\mathrm{X}$ & $\mathrm{X}$ & & \\
\hline $\begin{array}{l}\text { Temps } \\
\text { d'attente }\end{array}$ & $\mathrm{X}$ & $\mathrm{X}$ & & \\
\hline Absentéisme & $\mathrm{X}$ & & $\mathrm{X}$ & \\
\hline
\end{tabular}

Figure 5 Récapitulatif des outils de mesure présents dans les études

Nous constatons que seuls les outcomes « sexe », «MSQ ou autres » et «âge » se retrouvent dans les cinq études. Les autres outcomes ne sont présents que dans certaines études (quatre ou moins). C'est pourquoi nous séparons les résultats en trois parties, à savoir :

1) Résultats comparables aux cinq études [annexe 6]

2) Résultats comparables à quatre études [annexe 7]

\section{3) Autres résultats}

L'étude de Pendergast forme le $82.5 \%$ de l'échantillon total de nos résultats. Bien que ce nombre soit important et que l'étude n'aborde que trois outcomes, nous avons décidé de la garder et de traiter les données présentées dans les autres études. 
Ces dernières sont pertinentes et permettent d'aborder diverses thématiques concernant l'accès direct.

L'outcome «âge » se retrouve dans chaque étude mais n'est jamais catégorisé de la même manière. Il est présenté sous trois tranches d'âge différents rendant ainsi difficile une mise en commun des résultats. Cependant, nous avons tout de même décidé de le prendre en compte afin de répondre au mieux à notre question de recherche.

4.1 Résultats comparables aux cinq études

Les cinq études donnent un échantillon total de 76’000 personnes, à savoir 21'198 patients dans le groupe AD et 54'802 dans le groupe MG.

\section{Sexe}

\begin{tabular}{|c|c|c|c|c|}
\hline \multirow{2}{*}{} & \multicolumn{2}{c|}{ Accès direct } & \multicolumn{2}{c|}{ Médecin généraliste } \\
\cline { 2 - 5 } & $\mathrm{n}$ & $\%$ & $\mathrm{n}$ & $\%$ \\
\hline \multicolumn{4}{|c|}{ Données personnelles } \\
\hline Sexe & & & & 40.2 \\
\hline Homme & 8768 & 41.4 & 22025 & 59.8 \\
\hline Femme & 12430 & 58.6 & 32777 & \\
\hline
\end{tabular}

Figure 6 Tableau de l'outcome « Sexe »

Au total, les cinq études livrent un nombre d'hommes égal à 30'792 et un nombre de femmes égal à 45’208.

Dans le groupe AD, il y a 8'768 hommes, soit 41.4\% de l'échantillon, et 12’430 femmes, soit $58.6 \%$ de ce même échantillon.

Pour le groupe MG, les hommes sont présents pour un nombre total de 22'025, soit $40.2 \%$ de l'échantillon cité. Les femmes quant à elles, donnent un nombre de 32 ' 777 , soit $59.8 \%$.

Les résultats nous montrent deux groupes homogènes, si l'on considère le ratio homme/femme, environ $40 \%$ pour les hommes et environ $60 \%$ pour les femmes. En les comparant, nous voyons qu'il y a 1.2\% de plus d'hommes en $\mathrm{AD}$ et $1.2 \%$ de femmes en plus dans le groupe MG. Nous constatons qu'il n'y a donc pas de réelle différence et que les résultats pour cet outcome sont quasi semblables. 


\section{MSQ ou autres}

\begin{tabular}{|c|c|c|c|c|}
\hline \multirow{2}{*}{} & \multicolumn{2}{|c|}{ Accès direct } & \multicolumn{2}{c|}{ Médecin généraliste } \\
\cline { 2 - 5 } & $\mathrm{n}$ & $\%$ & $\mathrm{n}$ & $\%$ \\
\hline \multicolumn{4}{|c|}{ Données cliniques } \\
\hline Diagnostic & & & & \\
\hline MSQ & 17675 & 84.4 & 44236 & 81.2 \\
\hline $\begin{array}{c}\text { Autres (neuro, } \\
\text { uro,...) }\end{array}$ & 3275 & 15.6 & 10221 & 18.8 \\
\hline
\end{tabular}

Figure 7 Tableau de l'outcome « MSQ ou autre »

Plus de $98 \%$ de l'échantillon de base a répondu à cet outcome. Il manque $1.2 \%$ des données pour le groupe $\mathrm{AD}$ et $0.6 \%$ pour le groupe MG.

Cet outcome est séparé en deux catégories : «musculo-squelettique » et « autres », composée d'atteintes autre que MSQ, principalement neurologiques et urologiques.

Le total des diagnostics MSQ est égal à 61'911. Pour les autres diagnostics, le total est de 13'496.

En AD, les troubles MSQ sont diagnostiqués dans 17'675 cas, soit $84.4 \%$ de son échantillon. Les autres pathologies atteignent le nombre de 3'275 cas, soit $15.6 \%$.

Dans le groupe MG, le nombre de troubles MSQ diagnostiqués est égal à 44'236 cas, soit $81.2 \%$ de son échantillon. Les autres troubles donnent un total de 10 '221 cas, soit $18.8 \%$.

Nous constatons que les diagnostics MSQ sont présents à plus de 80\% dans les groupes $\mathrm{AD}$ et MG. La quantité est plus grande pour l'AD avec une différence de $3.2 \%$ par rapport au groupe MG.

\section{Age}

Comme expliqué plus haut, nous résumons cet outcome en tenant compte de la discussion des résultats de chaque étude.

Dans l'étude de Holdsworth parue en 2004, nous constatons que la tranche d'âge des 31-40 ans dans le groupe AD est plus représentée que dans le groupe MG. Par contre, la tranche d'âge 65-74 ans est plus grande dans le groupe MG. 
Les deux études du même auteur parues en 2005 ne montrent pas de réelles différences.

Dans l'étude de Leemrisje parue en 2008, nous constatons que les tranches d'âge 20-39 ans et 40-59 ans sont plus représentées dans le groupe AD que MG. Les personnes ayant 60 ans et plus sont par contre plus nombreuses dans le groupe MG.

Pendergast montre dans son étude de 2010 que la tranche d'âge de 18-24 ans est plus importante dans le groupe AD. A l'inverse, les 55-64 ans sont plus nombreux dans le groupe MG.

$\mathrm{Au}$ vu de ces résultats, nous remarquons que les patients en $\mathrm{AD}$ ont tendance à être plus jeunes que les patients consultant leur médecin au préalable.

4.2 Résultats comparables à quatre études

Les quatre études (Holdsworth 2004, 2005 (1), 2005 (2), Leemrisje 2008) donnent un échantillon total de 13'293 personnes, à savoir 3'701 dans le groupe $\mathrm{AD}$ et 9'592 dans le groupe MG.

\section{Diagnostics MSQ}

\begin{tabular}{|c|c|c|c|c|}
\hline \multirow{2}{*}{} & \multicolumn{2}{c|}{ Accès direct } & \multicolumn{2}{c|}{ Médecin généraliste } \\
\cline { 2 - 5 } & $\mathrm{n}$ & $\%$ & $\mathrm{n}$ & $\%$ \\
\hline \multicolumn{4}{|c|}{ Données cliniques } \\
\hline $\begin{array}{l}\text { Membre } \\
\text { supérieur }\end{array}$ & 276 & 12.5 & 872 & 18.5 \\
\hline Epaule & 228 & 82.6 & 698 & 80.0 \\
\hline Rachis & 1733 & 78.5 & 3327 & 70.5 \\
\hline Lombalgie & 733 & 42.3 & 1360 & 40.9 \\
\hline Cervicalgie & 682 & 39.4 & 1543 & 46.4 \\
\hline $\begin{array}{l}\text { Membre } \\
\text { inférieur }\end{array}$ & 199 & 9.0 & 523 & 11.1 \\
\hline Genou & 92 & 46.2 & 256 & 48.9 \\
\hline
\end{tabular}

Figure 8 Tableau pour l'outcome « MSQ ou autres »

Environ $70 \%$ de l'échantillon total a répondu à cet outcome. Il manque $24.9 \%$ de diagnostics dans le groupe $\mathrm{AD}$ et $32.1 \%$ dans le groupe $\mathrm{MG}$.

Afin de pouvoir mieux visualiser les résultats, nous avons décidé de les séparer par région du corps : membre supérieur, rachis et membre inférieur. 


\section{$\underline{\text { Membre supérieur }}$}

Les quatre études regroupent 1'148 cas touchant cette partie du corps. 276 patients en $\mathrm{AD}$ et 872 en MG. Cela représente 12.5\% de l'échantillon des patients en $\mathrm{AD}$ et $18.5 \%$ des patients référés par leur médecin.

Les pathologies touchant l'épaule sont les plus fréquentes dans les quatre études. 228 cas en $\mathrm{AD}$, soit $82.6 \%$ de l'échantillon membre supérieur $\mathrm{AD}$, et 698 cas dans le groupe MG, soit 80\%. Les autres diagnostics du membre supérieur ne sont pas détaillés.

\section{$\underline{\text { Rachis }}$}

Les quatre études regroupent 5'060 cas touchant le rachis. 1'733 patients en $\mathrm{AD}$ et 3’327 en MG. Cela représente $78.5 \%$ de l'échantillon des patients en $\mathrm{AD}$ et $70.5 \%$ des patients MG.

La plupart des cas traités dans les quatre études concernent surtout les lombalgies et les cervicalgies. En AD, 733 lombalgies, soit $42.3 \%$ de l'échantillon Rachis, et 682 cervicalgies, soit 39.4\%, sont traitées. Dans le groupe MG, 1'360 lombalgies, soit 40.9\% de 1'échantillon Rachis, et 1'543 cervicalgies, soit $46.4 \%$, sont prises en charge. Les autres diagnostics du rachis ne sont pas détaillés.

\section{$\underline{\text { Membre inférieur }}$}

Les quatre études regroupent 722 cas touchant cette région du corps. 199 patients en AD et 523 en MG. Cela représente 9\% de l'échantillon des patients en $\mathrm{AD}$ et $11.1 \%$ des patients MG.

Les pathologies touchant le genou sont les plus fréquentes dans les quatre études. 92 cas, soit $46.2 \%$ de l'échantillon membre inférieur, sont vus par leur physiothérapeute en AD. Le groupe MG comporte 256 cas, soit 48.9\%. Les autres diagnostics du membre inférieur ne sont pas détaillés.

$\mathrm{Au}$ vu de tous ces résultats, nous remarquons que les pathologies du rachis sont les plus représentées, surtout par les régions lombaire et cervicale. Les patients souffrant de tels maux auraient une tendance plus marquée à se rendre chez leur physiothérapeute en $\mathrm{AD}$ (78.5\% contre $70.5 \%$ en MG). 


\section{Durée des symptômes}

\begin{tabular}{|c|c|c|c|c|}
\hline \multirow{2}{*}{} & \multicolumn{2}{c|}{ Accès direct } & \multicolumn{2}{c|}{ Médecin généraliste } \\
\cline { 2 - 5 } & $\mathrm{n}$ & $\%$ & $\mathrm{n}$ & $\%$ \\
\hline \multicolumn{4}{|c|}{ Données cliniques } \\
\hline Durée des symptômes & & & & \\
\hline$<1$ mois & 1733 & 47.4 & 3167 & 33.6 \\
\hline 1 - 3 mois & 748 & 20.5 & 2209 & 23.5 \\
\hline$>3$ mois & 1175 & 32.1 & 4042 & 42.9 \\
\hline
\end{tabular}

Figure 9 Tableau de l'outcome « Durée des symptômes »

Plus de $98 \%$ de l'échantillon de base a répondu à cet outcome. Il manque seulement $1.3 \%$ des données pour le groupe AD et $1.9 \%$ pour le groupe MG.

Afin de pouvoir mieux visualiser les résultats, nous avons décidé de les séparer en trois groupes (inférieur à un mois, de un à trois mois, supérieur à trois mois). Dans chaque étude, la durée des symptômes est mesurée dès l'apparition de ces derniers (pré-consultation).

L'échantillon total est composé de 13'074 personnes, dont 3'656 consultent leur physiothérapeute en $\mathrm{AD}$ et 9’418 vont chez leur médecin.

\section{$\underline{\text { Inférieur à un mois }}$}

Dans les quatre études, le nombre de personnes présentant des symptômes dont la durée est inférieure à un mois est égal à 4’900.

1'733 patients du groupe $\mathrm{AD}$ ont souffert moins d'un mois de leurs symptômes, ce qui représente $47.4 \%$ de son échantillon. Pour le groupe MG, 3'167 personnes ont ressenti leurs symptômes durant cette durée, soit 33.6\% de l'échantillon MG.

\section{$\underline{\text { De un à trois mois }}$}

Dans les quatre études, le nombre de personnes présentant des symptômes dont la durée va de un à trois mois est égal à 2'957.

748 patients du groupe $\mathrm{AD}$ ont souffert de un à trois mois de leurs symptômes, ce qui représente $20.5 \%$ de son échantillon. Pour le groupe MG, 2’209 personnes ont ressenti leurs symptômes durant cette durée, soit $23.5 \%$ de l'échantillon MG. 


\section{$\underline{\text { Supérieur à trois mois }}$}

Dans les quatre études, le nombre de personnes présentant des symptômes dont la durée est supérieure à trois mois est égal à 5’217.

1'175 patients du groupe AD ont souffert pendant plus de trois mois de leurs symptômes, ce qui représente $32.1 \%$ de l'échantillon AD. Pour le groupe MG, 4'042 personnes ont eu leurs symptômes durant cette durée, soit 42.9\% de l'échantillon MG.

$\mathrm{Au}$ vu de ces résultats, nous pouvons constater que la majorité des patients en $\mathrm{AD}$, soit $47.4 \%$ des cas, ont une durée de symptômes inférieure à un mois, contre $33.6 \%$ des cas dans le groupe MG. Par contre, un pourcentage non négligeable de patients qui consultent leur médecin au préalable ont des symptômes qui durent plus de trois mois (42.9\% contre $32.1 \% \mathrm{AD})$.

\section{Arrêt du TTT}

\begin{tabular}{|c|c|c|c|c|}
\hline \multirow{2}{*}{} & \multicolumn{2}{|c|}{ Accès direct } & \multicolumn{2}{c|}{ Médecin généraliste } \\
\cline { 2 - 5 } & $\mathrm{n}$ & $\%$ & $\mathrm{n}$ & $\%$ \\
\hline \multicolumn{4}{|c|}{ Données cliniques } \\
\hline Arrêt du TTT & & & & \\
\hline TTT complet & 1759 & 83.6 & 4477 & 75.4 \\
\hline $\begin{array}{c}\text { TTT incomplet ou } \\
\text { arrêté }\end{array}$ & 189 & 9.0 & 809 & 13.6 \\
\hline Autre & 156 & 7.4 & 651 & 11.0 \\
\hline
\end{tabular}

Figure 10 Tableau de l'outcome « Arrêt du TTT »

Cet outcome à réuni $60 \%$ des données de l'échantillon total. Il manque $43.2 \%$ de résultats pour le groupe $\mathrm{AD}$ et $38.1 \%$ pour le groupe $\mathrm{MG}$.

Afin de pouvoir mieux comparer les résultats entre eux, nous avons décidé de garder les trois catégories qui se retrouvaient dans les quatre études, à savoir : TTT complet, TTT incomplet ou arrêté, autre.

L'échantillon total est composé de 8'041 personnes, dont 2'104 patients AD et 5'937 patients MG. 


\section{$\underline{\text { TTT complet }}$}

Dans les quatre études, le nombre de personnes ayant terminé leur TTT est égal à 6’236.

1'759 patients du groupe $\mathrm{AD}$ ont mené à terme leur TTT, ce qui représente 83.6\% de son échantillon. Pour le groupe MG, 4’477 ont terminé leur TTT, soit 75.4\% de l'échantillon MG.

\section{$\underline{\text { TTT incomplet ou arrêté }}$}

Dans les quatre études, le nombre de personnes n'ayant pas terminé leur TTT ou l'ayant arrêté est égal à 998.

189 patients du groupe $\mathrm{AD}$ n'ont pas mené à terme leur TTT, ce qui représente 9\% de son échantillon. Pour le groupe MG, 809 n'ont pas terminé leur TTT, soit 13.6\% de l'échantillon MG.

\section{$\underline{\text { Autre }}$}

Ce groupe comprend les patients retournés chez leur médecin, le TTT interrompu après une séance, ainsi que les données manquantes dans les quatre études. Le nombre est égal à 807.

156 patients du groupe $\mathrm{AD}$ se retrouvent dans ce groupe, ce qui représente 7.4\% de son échantillon. Pour le groupe MG, 651 patients sont répertoriés dans ce groupe, soit $11 \%$ de l'échantillon MG.

A l'aide de ces résultats, nous remarquons que les patients du groupe AD ont terminé leur traitement dans $83.6 \%$ des cas, contre $75.4 \%$ dans le groupe MG. C'est la seule remarque que nous pouvons faire, étant donné que les autres catégories (TTT incomplet ou arrêté et autre) ne sont pas détaillées de manière précise dans les études. 


\section{Réussite des objectifs}

\begin{tabular}{|c|c|c|c|c|}
\hline \multirow{2}{*}{} & \multicolumn{2}{|c|}{ Accès direct } & \multicolumn{2}{c|}{ Médecin généraliste } \\
\cline { 2 - 5 } & $\mathrm{n}$ & $\%$ & $\mathrm{n}$ & $\%$ \\
\hline \multicolumn{5}{|c|}{ Données cliniques } \\
\hline Réussite des objectifs & & & & \\
\hline $0 \%$ & 52 & 3.1 & 301 & 6.5 \\
\hline $25-75 \%$ & 362 & 21.9 & 1222 & 26.4 \\
\hline $100 \%$ & 1237 & 74.9 & 3107 & 67.1 \\
\hline
\end{tabular}

Figure 11 Tableau de l'outcome « Réussite des objectifs »

Moins de 50\% des patients de l'échantillon total ont répondu à cet outcome. Il manque $55.4 \%$ des données pour le groupe $\mathrm{AD}$ et $51.7 \%$ pour le groupe MG.

Afin de mieux pouvoir comparer les résultats entre eux, nous avons décidé de garder les catégories qui se retrouvaient dans les quatre études, à savoir : 0\%, $25-75 \%$ et $100 \%$ de réussite.

L'échantillon total est composé de 6'281 personnes, dont 1'651 patients AD et 4'630 patients MG.

\section{$\underline{0 \% \text { de réussite }}$}

Dans les quatre études, le nombre des personnes ayant atteint $0 \%$ de réussite dans leur TTT est de 353.

52 patients du groupe AD disent ne pas avoir atteint l'objectif thérapeutique, ce qui représente $3.1 \%$ de son échantillon. Pour le groupe MG, 301 patients n'ont pas réussi leur TTT, soit 6.5\% de l'échantillon MG.

\section{5-75\% de réussite}

Dans les quatre études, le nombre de personnes ayant atteint entre 25 et $75 \%$ de réussite dans leur TTT est de 1'584.

362 patients du groupe $\mathrm{AD}$ disent avoir atteint partiellement l'objectif thérapeutique, ce qui représente $21.9 \%$ de son échantillon. Pour le groupe MG, cela donne 1'222 personnes, soit 26.4\% de l'échantillon MG.

$100 \%$ de réussite

Dans les quatre études, le nombre de personnes ayant obtenu un résultat de $100 \%$ suite à leur TTT est de 4’344. 
1'237 patients du groupe AD disent avoir atteint entièrement l'objectif thérapeutique, ce qui représente $74.9 \%$ de son échantillon. Pour le groupe MG, cela donne 3'107 personnes, soit 67.1\% de 1'échantillon MG.

Suite à ces résultats, nous remarquons que le taux de réussite à 100\% est plus élevé dans le groupe $\mathrm{AD}$ que dans le groupe MG (74.9\% contre 67.1\%). La catégorie «25-75\% » est trop vaste pour en tirer une conclusion et n'est donc pas pertinente dans la pratique quotidienne d'un physiothérapeute.

\subsection{Autres résultats}

Cette partie des résultats est sans doute la moins pertinente vu le manque de données et de points de comparaison. Il faudrait donc les relativiser. Mais les thèmes abordés et les informations qui en ressortent sont intéressants ; il serait dommage de ne pas les citer.

\section{Zone géographique du cabinet}

Leemrisje (2008) présente dans son étude cinq catégories d'urbanisation : very strong, strong, moderate, little et not. Holdsworth 2005 (1) différencie trois types de zone :

1) Rurale : communauté composée de moins de 3'000 habitants, incluant les frontières, les montagnes et les îles. Activité principale : travail de la terre.

2) Semi-urbaine: densité de population comprise entre 200 et 499 habitants $/ \mathrm{km}^{2}$.

3) Urbaine : zone étendue jusqu'à trente minutes de temps de trajet du centre de la ville.

Pour simplifier la lecture de ces résultats et pour les comparer au mieux, nous avons décidé de garder le modèle de Holdsworth en réduisant les catégories de Leemrisje à trois : very strong et strong pour urbain, moderate et little pour semi-urbain et not pour rurale.

Au total, les deux études regroupent soixante-neuf cabinets de physiothérapie. Neuf d'entre eux se trouvent en zone rurale, vingt-cinq en zone semi-urbaine et trente-cinq en région urbaine. 
Nous pouvons tirer de ces chiffres une tendance à une localisation plus dense des cabinets de physiothérapie en milieu urbain. D'après ces résulats, un cabinet sur sept se trouverait en zone rurale et un cabinet sur deux en ville.

\section{Niveau de formation du patient}

Seule Leemrisje (2008) étudie cet outcome. Après avoir récolté les données personnelles des participants de l'étude, elle a classé leur niveau de formation en trois divisions : éducation basse, moyenne et haute. L'auteur ne définit pas à quels niveaux scolaires correspondent ces trois catégories.

Nous constatons, d'après l'étude, que les personnes ayant un bas niveau de formation sont plus représentées dans le groupe MG et celles avec un niveau moyen dans le groupe AD. Les patients ayant un haut niveau de formation auraient tendance à se rendre plus facilement chez leur physiothérapeute en AD.

\section{Statut professionnel}

Cet outcome est mesuré dans deux études de Holdsworth (2004 et 2005 (2)). Dans son étude de 2004, elle regroupe trois catégories : employé, retraité et autre (au foyer, chômage, étudiant). Celle de 2005 (2) comporte cinq groupes : employé, retraité, au foyer, chômage et étudiant. Vu que nous retrouvons les mêmes divisions, nous avons décidé de mettre ensemble les catégories «au foyer », «chômages » et «étudiant» sous le nom «autre», à l'instar de l'auteur dans son étude de 2004.

Des résultats que nous obtenons, nous pouvons essentiellement dégager le fait que ce sont surtout les personnes employées ou à la retraite qui consultent leur médecin ou leur physiothérapeute. Les résultats entre les deux groupes sont par ailleurs quasi similaires.

\section{Temps d'attente}

Comme pour l'outcome précédent, seule Holdsworth prend en compte cet aspect de la prise en charge dans ses deux études de 2004 et 2005 (2).

Après constat, nous voyons que le temps d'attente d'une durée de un à trois mois est quasi semblable pour les deux groupes. Néanmoins, un patient 
semblerait avoir plus facilement un rendez-vous dans un intervalle de deux semaines chez son physiothérapeute que chez son généraliste.

\begin{abstract}
Absentéisme
Comme pour les deux outcomes précédents, seule Holdsworth parle de ce sujet dans ses études de 2004 et 2005 (2).

Les personnes consultant leur physiothérapeute en $\mathrm{AD}$ seraient en grande majorité absents de leur travail moins de deux semaines, alors que les patients du groupe MG seraient absents d'une semaine à un mois. Cependant dans les deux études, l'auteur a montré que le taux d'absentéisme parmi les personnes employées est relativement bas. Les valeurs restent donc faibles et nous obligent à les considérer avec une certaine distance.
\end{abstract}




\section{Discussion}

5.1 Interprétation des résultats

Le but de notre travail est de déterminer s'il existe une différence entre le profil d'un patient consultant son physiothérapeute en accès direct et celui qui sollicite son médecin au préalable. Après avoir exposé les résultats de manière brute, voici ce que nous en retirons.

\section{Résultats comparables aux cinq études}

L'échantillon pour la catégorie «sexe» comporte plus de femmes que d'hommes. Les pays en question ont aussi une population féminine plus étoffée que la population masculine, comme l'expliquent les auteurs. Cependant, nos résultats montrent peu de différences entre le groupe $\mathrm{AD}$ et le groupe MG. Nous ne pouvons donc pas mettre en avant un genre pour répondre à notre question de profil type.

Les résultats de l'outcome «MSQ ou autres » révèlent la prédominance des pathologies musculo-squelettiques, tant en consultation physiothérapeutique que médicale. Ce qui reflète la réalité tout comme l'OFS le démontre en Suisse dans ses rapports en 2011. Elle indique aussi que les femmes ont statistiquement plus de problèmes MSQ que les hommes.

Suite à notre analyse sur les âges, les résultats montrent que les patients en $\mathrm{AD}$ sont plus jeunes que les patients allant consulter leur médecin. Pour la population, le médecin reste la première personne à consulter en cas de maladie. C'est surtout le cas chez les personnes âgées qui entretiennent depuis longtemps une relation de confiance avec leur médecin de famille, considéré comme étant le spécialiste de la santé. La nouvelle génération peut accéder plus facilement à diverses sources d'informations comme internet; elle a donc moins de difficulté à aller directement chez son physiothérapeute. Cette génération est plus impliquée dans le maintien de sa santé et a la capacité de se diriger en cas de besoin vers le professionnel adéquat.

L'AD offre une possibilité de parcours de soin supplémentaire au patient. Si elle avait existé depuis de nombreuses années, comme c'est le cas en Australie, elle serait certainement entrée dans les mœurs. Chaque génération connaîtrait 
alors l'existence d'un tel procédé et ne se poserait peut-être plus la question de savoir vers quel professionnel de la santé se tourner, selon les besoins.

\section{Résultats comparables à quatre études}

En précisant maintenant le diagnostic MSQ, l'analyse montre que ce sont principalement les pathologies rachidiennes qui sont citées dans nos études. Cela ne nous étonne guère, dans le sens où le «mal de dos » est considéré comme le «mal du siècle » et que les campagnes de promotion et prévention de la santé le mettent en avant comme tel. Les lombalgies sont plus nombreuses en $\mathrm{AD}$. Peut-être à cause de la nature du problème : une lombalgie, idiopatique ou non-spécifique, peut s'améliorer grâce à la physiothérapie ; le patient a certainement envie d'être traité manuellement et c'est pour cela qu'il consulte son physiothérapeute. Mais le diagnostic cervicalgie est plus fréquent dans le groupe MG. Ceci est peut-être dû à la nature du problème. La colonne cervicale est une région sensible et il est facile pour un patient d'imaginer le pire (tétraplégie, pathologies crânienes, etc). Les patients préfèrent peut-être consulter alors leur médecin. Dans le groupe MG, les pathologies touchant les membres sont plus nombreuses qu'en AD. Le genou et l'épaule sont les plus fréquemment cités. Sachant que ce sont des articulations en général très sollicitées et qu'elles sont sujettes à des traumatismes ou des dégénérescences, des examens médicaux supplémentaires sont souvent indispensables et nécessitent donc une visite chez le médecin.

Les résultats sur la durée des symptômes montrent que près de $50 \%$ des patients en $\mathrm{AD}$ sont algiques moins d'un mois. Par contre, $42.9 \%$ des patients consultant leur médecin au préalable ont des douleurs qui durent plus de trois mois. La différence est de taille. On peut l'expliquer par le fait que le patient décide d'aller directement chez son physiothérapeute - ce qui prouve une certaine motivation. Il y va et veut être actif lors de son traitement et dans sa convalescence. Le physiothérapeute éduque aussi le patient. Il lui fait comprendre qu'il doit s'investir durant et en dehors de la séance pour permettre une évolution favorable. Une deuxième explication réside dans le résultat de l'outcome «temps d'attente» de la catégorie autres résultas. Le temps d'attente pour un rendez-vous chez son physiothérapeute est plus court que pour un rendez-vous chez son médecin. Il semble donc logique d'admettre que 
les symptômes disparaissent plus rapidement si le patient est plus vite pris en charge. N'oublions pas la qualité de TTT du PT, qui peut jouer aussi un rôle.

L'outcome «arrêt du TTT » montre que $83.6 \%$ des patients en AD finissent leur traitement contre $75.4 \%$ dans le groupe MG. L'analyse de l'outcome « réussite des objectifs» montre aussi une différence entre les deux groupes. Pour un résultat d'objectifs atteints à $100 \%$, nous trouvons $74.9 \%$ des patients AD contre $67.1 \%$ des patients MG. Nous pensons que la prise en charge et la motivation du patient influencent ces résultats. Le patient se prend en charge et se dirige de son propre chef vers le thérapeute compétent, contrairement au patient MG qui est adressé au physiothérapeute par son médecin. Il paraît donc normal qu'il décide d'aller au bout de sa thérapie et qu'il mette tout en œuvre pour atteindre ses objectifs.

\section{Autres résultats}

Selon les autres résultats que nous avons recueillis, nous pouvons en tirer les conclusions suivantes :

L'outcome «zone géographique du cabinet » démontre que la grande majorité des cabinets de physiothérapie se trouvent en ville - tout comme la population actuelle. Néanmoins, il reste tout de même des cabinets en zones rurales, ce qui permet de garantir des soins de premier recours aux personnes habitant ces régions. Nous reviendrons sur ce sujet un peu plus loin dans la discussion.

En ce qui concerne le niveau d'éducation des patients, nous pouvons le mettre en relation avec l'outcome «âge » cité plus haut. Les patients du groupe AD présentent, d'après Leemrisje, un niveau de formation supérieur aux patients $d u$ groupe MG. Ce fait est lié aux sources d'informations à disposition des personnes, informations qui leur permettent d'avoir de meilleures connaissances sur leur état de santé (anatomie, physiologie) et sur les différents thérapeutes susceptibles de traiter le mal en question. Le patient d'aujourd'hui ne ressemble plus à celui d'hier : il est proactif dans son TTT.

Le statut professionnel montre que les personnes employées ou retraitées consultent un médecin ou un physiothérapeute. Il n'y a pas de différence notable entre les deux groupes, excepté une légère tendance à retrouver plus de retraités dans le groupe MG (comme développé plus haut sur la relation 
patient/médecin pour l'ancienne génération) et plus d'employés dans le groupe AD. Y aurait-il un désir de gagner du temps et de pouvoir retourner au plus vite au travail ?

En considérant l'absentéisme, nous avons remarqué que le taux était relativement bas dans les deux groupes. Ce résultat est conforme à l'opinion suisse issue du sondage effectué par l'agence gfs.bern en 2009 : 81\% des 1014 Suisses de l'étude ont répondu «oui » à la question : «Pensez-vous qu'un traitement physiothérapeutique après une interruption de travail suite à un accident ou une maladie permette de revenir plus tôt à son poste de travail ?» (Monnin, 2011, p. 213). Néanmoins, les personnes consultant leur physiothérapeute en $\mathrm{AD}$ seraient moins absentes à leur travail que les personnes du groupe MG. Comme déjà expliqué plus haut, ceci est peut-être dû au fait que les patients ont le choix d'aller directement chez leur physiothérapeute, par désir ou par besoin de retourner plus vite au travail.

Forts de ces résultats, nous pouvons répondre à notre premier objectif. Le profil d'un patient en $\mathrm{AD}$ est différent de celui d'un patient MG. Ils se différencient principalement sur les points suivants :

1) Âge

2) Diagnostic MSQ

3) Durée des symptômes

4) Raison arrêt du TTT

5) Réussite des objectifs

Holdsworth dans ses études de 2004 et 2005 (2) ainsi que Leemrisje (2008), montrent aussi d'autres disparités concernant le niveau de formation des patients, le temps d'attente pour un rendez-vous et l'absentéisme. 
Notre deuxième objectif est de définir un profil type de patient consultant son PT en accès direct. Grâce à nos résultats, il est possible de faire ressortir une donnée personnelle et une donnée clinique. Le profil type d'un patient consultant son physiothérapeute en AD :

1) serait une personne âgée de moins de quarante ans

2) souffrant d'une pathologie d'origine musculo-squelettique concernant la plupart du temps le rachis.

Leemrisje (2008) démontre aussi que les patients en $\mathrm{AD}$ ont un niveau de formation moyen à supérieur.

\subsection{La physiothérapie et l'AD en 2013}

La profession d'aujourd'hui n'est pas la même que celle d'hier. Les physiothérapeutes ne sont plus de simples exécutants qui appliquent des techniques peu différenciées. La physiothérapie a acquis au fil du temps des compétences spécifiques que d'autres professions ne maîtrisent pas, comme la capacité de choisir les tests spécifiques permettant de différencier l'origine d'un trouble, d'évaluer l'efficacité du TTT selon la succession «test-treattest » et de l'adapter si besoin, la sensibilité du toucher (« les yeux au bout des doigts »), la relation de confiance physiothérapeute/patient, etc. Cette profession touche énormément de domaines. Elle ne cesse d'évoluer, grâce notamment à la recherche EBM, qui permet de prouver l'efficacité de nos TTT.

En Suisse, les physiothérapeutes souhaitent obtenir plus de reconnaissance de la part des médecins, des politiciens, des assurances et des patients. Ils veulent être reconnus à leur juste valeur, comme étant des professionnels de la santé qui ont une formation de base solide, qui suivent des formations continues pour maintenir leurs connaissances à jour et qui garantissent une prise en charge de qualité, tout en étant payés de manière décente.

Les discussions autour de l'accès direct en Suisse sont nombreuses. Certains le voient comme un moyen supplémentaire de reconnaissance. D'autres comme l'évolution logique de notre profession, selon ce que l'on constate dans les pays voisins. Rappelons que la formation en Suisse offre un titre de «Bachelor of Science in Physiotherapy » après trois ans d'étude, tout comme au Royaume-Uni, en Suède et aux Pays-Bas (CDS-CRS, 2003, pp. 72-76), qui eux proposent en outre un $\mathrm{AD}$ en physiothérapie pour soins publics ou privés (Bury 
\& Stokes, 2012). D'autres voient encore l'AD comme un moyen de pallier à la pénurie annoncée de médecins généralistes. En effet, certains fonds de vallée et certains villages sont de véritables déserts à $\mathrm{MG}$. Un physiothérapeute en $\mathrm{AD}$ permettrait aux patients d'avoir un premier contact médical supplémentaire et de soulager en partie le travail des MG. D'ailleurs, en Valais, une commission composée de divers acteurs du monde de la santé a été mandatée par le département de la santé en 2013. Celle-ci aura pour mission d'analyser la situation du canton en matière de soins ambulatoires, de premiers recours et de trouver des solutions concrètes en cas de besoin (Canton du Valais, 2013).

Et que pensent les physiothérapeutes suisses de l'accès direct? Dans le sondage de l'agence gfs.bern mené en $2009,82 \%$ des physiothérapeutes suisses participant à l'étude sont favorables à l'accès direct et $80 \%$ se sentent prêts à travailler dans ces conditions. Notons juste que $71 \%$ des participants à ce sondage avaient plus de 10 ans d'expérience professionnelle. $57 \%$ pensent tout de même que travailler en accès direct demanderait des qualifications supplémentaires, notamment dans les « red flags » et le diagnostic différentiel, et supposerait la participation à des formations continues (Allet, 2011, pp. 22531). Fort de ce sondage, un projet pilote d'AD en physiothérapie mandaté par Physioswiss et sous la direction de la HES Zürich devrait voir le jour. Vu les aspects qu'ils pensent prendre en compte et évaluer, ce projet devrait s'inscrire pleinement dans la dynamique actuelle de réduction des coûts et de recherche d'alternatives. Ce qui, en cas de résultats positifs, donnerait du poids aux physiothérapeutes face aux politiques.

\section{Avantages versus risques}

La littérature des pays dans lesquels l'accès direct en physiothérapie est en vigueur cite principalement les avantages de cette modalité de parcours de soins. La littérature cite (Scheermesser, 2011, pp. 220-1) :

1) Des soins rapides et adéquats, grâce à la diminution du temps d'attente par rapport à un médecin, et à la qualité de la prise en charge. Ceci entraîne une satisfaction des patients et des physiothérapeutes.

2) Une collaboration entre les professionnels de la santé améliorée, avec une relation de confiance renforcée, ainsi qu'une aide et un soulagement pour le travail des MG. 
3) La gestion efficace des ressources financières, grâce notamment à la réduction des prescriptions de médicaments, des clichés médicaux $(\mathrm{Rx}$, IRM, etc) et du nombre de thérapeutes à consulter.

Après des recherches sur différentes bases de données, nous pouvons évoquer certains risques en défaveur de l'accès direct :

1) Le diagnostic médical : comparé à un médecin, le physiothérapeute a moins de connaissances et de moyens techniques (Rx, IRM, US, etc.) pour diagnostiquer une pathologie. Il ne peut pas faire de bilan aussi poussé et précis ; il serait possible qu'il passe à côté de contre-indications importantes pouvant mettre en danger la santé du patient.

2) Décision de management : ce risque est lié au précédent. Après avoir fait son bilan, le PT peut traiter directement son patient, le traiter avec un avis médical ou ne pas le traiter et le rediriger vers un médecin. Une mauvaise décision, liée à un mauvais diagnostic, peut mettre en danger le patient.

Nous pensons aussi à d'autres risques, comme la surdemande ou la faible demande d'AD. Cependant, la littérature ne semble pas en montrer à ce niveau là. Par contre pour les risques de diagnostic médical et de management, Moore dans son étude de 2005, Jette (2006) et Ludvigsson (2011) déclarent que les physiothérapeutes en $\mathrm{AD}$ ont plus de facilité à poser un diagnostic lorsque la pathologie est non-critique et d'origine MSQ, mais aussi qu'ils prennent la bonne décision de management lorsqu'il s'agit d'une pathologie critique, à savoir rediriger immédiatemment le patient vers un médecin compétent.

\section{Perspectives professionnelles et défis futurs}

Nous nous rendons compte que la physiothérapie a beaucoup évolué ces dernières années. Comme nous l'avons cité précédemment, les physiothérapeutes suisses espèrent plus de reconnaissance. Certaines modifications pourraient y mener, telle que la prescription médicale qui ressemble parfois plus à une ordonnance, limitant le physiothérapeute dans ses actions thérapeutiques et son raisonnement. Celle-ci pourrait ne comprendre que les données administratives du patient, le(s) diagnostic(s) et les contreindications, lui laissant une pleine liberté dans son TTT. Cette modification serait déjà un signe de reconnaissance et de confiance de la part des médecins. 
Dans un autre domaine, une augmentation de la valeur du point de physiothérapie serait un signe de gratitude des politiciens. Un temps de travail «administratif» (rédaction de rapport et de bilan, de remarques et de TTT, appel téléphonique, recherche) rémunéré, comme les médecins et les ergothérapeutes, serait aussi un grand pas pour notre profession. Sans oublier, une reconnaissance salariale pour les physiothérapeutes selon leur niveau de formation (CAS, DAS, MAS, MSc, PhD), ce qui n'est actuellement pas le cas.

Pourquoi dès lors ne pas proposer un $\mathrm{AD}$ en physiothérapie qui s'inscrit pleinement dans cette volonté de reconnaissance des physiothérapeutes suisses ? Cette question mérite encore de la réflexion et devrait passer par plusieurs étapes: un projet pilote qui montre des résultats avantageux (prochainement), un projet pilote au niveau national (si nécessaire), une demande auprès des autorités supérieures, une modification des textes de loi (Boldi-Goetshy, 2011, pp.235-37), notamment dans la LAMal et l'OAMal pour définir les physiothérapeutes comme fournisseurs de prestation en $\mathrm{AD}$ et dans l'OPAS, pour que l'AD en physiothérapie soit remboursé par la caisse maladie de base, etc. Personnellement, nous estimons, en tant qu'étudiants, que la formation de base devrait être plus poussée en ce qui concerne le diagnostic différentiel et la connaissance des « red flags ». Si toutes ces conditions étaient réunies, nous pensons qu'un accès direct en physiothérapie serait envisageable en Suisse.

\subsection{Limitations du travail}

Une première limitation survient dans la définition du profil patient de notre cadre théorique. N'ayant pas trouvé de sources scientifiques détaillant de manière précise ce terme, nous avons rédigé une définition par nos propres moyens, en nous inspirant d'études ou de discussions sur l'accès direct.

Nous pouvons également citer le fait que nous n'avons pas trouvé, dans chaque pays ayant l'accès direct en physiothérapie, des articles répondant à nos objectifs et question de recherche. Il nous manque l'Australie, le Canada, la Suède et la Norvège pour faire une étude comparative optimale.

Parlons de notre échelle de qualité. La version de base de l'échelle de Law parle dans l'item intervention de «contamination » et de «cointervention», 
aspects qui ne sont pas traités dans les études que nous avons sélectionnées. L'intervention elle-même n'est pas détaillée. C'est pourquoi nous avons adapté notre barème après avoir décidé de quantifier chaque item, ceci par simplification, car nous trouvions plus adéquat d'avoir une valeur chiffrée plutôt qu'une valeur subjective. Mais cette quantification qui ne tient pas compte des deux items susmentionnés engendre une modification de la version de base - ce qui entraîne un biais dans cette évaluation de la qualité.

Lorsque nous avons effectué l'extraction de données, les études ne comportaient pas toujours les mêmes outcomes ou elles ne les comptabilisaient pas de la même manière. Nous avons donc dû modifier notre grille d'extraction selon l'étude ou adapter les catégories pour permettre une comparaison.

Enfin, une autre limitation intervient lors du calcul de l'échantillon de base. En effet, nous nous sommes rendus compte que l'étude de Pendergast englobait le $82,5 \%$ de l'échantillon total des patients. Ainsi, les résultats des outcomes comparables dans les cinq études sont grandement influencés par ceux propres à l'étude de Pendergast. Certaines fois, l'échantillon de base des patients dans les trois études de Holdsworth et celle de Leemrisje ne correspondaient pas au nombre de cas présents par outcome. Il y avait soit des «missing data », soit des « disparitions » sans explication.

5.4 Recommandations futures

Il serait intéressant d'avoir plus d'informations sur la profession des patients, car ce qui est proposé dans les études de Holdsworth ne concerne que les statuts «employé », « retraité » et « autre ». S'il y avait cette précision, nous pourrions peut-être trouver un lien entre le niveau de formation d'une personne, le domaine professionnel et le fait qu'elle aille chez son physiothérapeute en $\mathrm{AD}$ ou non.

Nous trouverions pertinent d'avoir également des études qui précisent pour chaque articulation leur fréquence d'apparition. Une « cartographie » précise du corps humain décrirait les articulations les plus traitées en physiothérapie et permettrait aux physiothérapeutes de maintenir à jour leurs connaissances sur les pathologies les plus fréquentes. 


\section{Conclusion}

Ce travail de Bachelor est orienté sur un aspect moins connu de la profession, ce qui lui confère son originalité. Du fait que l'accès direct en physiothérapie existe ou débute dans plusieurs pays, il fait partie de l'actualité et touche directement les physiothérapeutes. Ce thème a d'ailleurs déjà été abordé lors de différents congrès nationaux en Suisse et le sera encore certainement à l'avenir.

Notre revue montre que le profil des personnes consultant leur médecin avant d'aller chez un physiothérapeute est différent de celui venant chez leur PT en accès direct. Il existe des divergences dans l'âge, le diagnostic, la durée des symptômes, le taux de réussite des objectifs et le temps d'attente pour un rendez-vous. Le profil type d'une personne du groupe $\mathrm{AD}$ se démarque par une donnée personnelle - l'âge - et une donnée clinique - le diagnostic.

Les résultats de ce travail démontrent que l'accès direct en physiothérapie ne change en rien la qualité de prise en charge des patients et qu'il répond à une demande. L'accès direct pourrait valoriser notre profession, soulager le travail des médecins généralistes et permettre des soins de premiers recours dans les zones reculées.

Reste à déterminer maintenant, au moyen d'études et de projets pilotes en Suisse, la qualité de l'accès direct et son impact précis sur les coûts de la santé. S'il en ressort des conclusions positives et que les différents acteurs de la santé s'accordent, l'accès direct en Suisse pourrait être envisageable. 


\section{Références bibliographiques}

Bury, J., \& Stokes, E.K. (2012). A Global View of Direct Access and Patient SelfReferral to Physical Therapy: Implication for the Profession. Physical Therapy, 93, 449-459. doi: 10.2522/ptj.20120060

Canton du Valais. (2013). Commission d'expert «Soins ambulatoires et de premier recours »-Mandat de la commission. Département des finances, des institutions et de la santé du canton du Valais, Sion.

Confédération suisse. (2013). Statistique suisse. Accès http://www.bfs.admin.ch/bfs/portal/fr/index/themen/14/05/blank/key/ueberblick. html

Confédération suisse. (2013). Statistique suisse. Accès http://www.bfs.admin.ch/bfs/portal/fr/index/themen/14/04/01/key/diagnosen.htm 1

Conférence des directeurs cantonaux des affaires sanitaires, Croix-Rouge suisse. (2003). Formations des diplômes en santé - aperçu général [Document de travail] (pp. 72-76). Bern : Conférence des directeurs cantonaux des affaires sanitaires.

Crout, K.L., Hodgkins, J., \& Miller T.D. (1998). Physical Therapists' Opinions and Practices Regarding Direct Access. Physical Therapy, 78, 52-61.

Fédération des Médecins Suisses. (2013). Statistique médicale de la FMH. Accès http://www.fmh.ch/fr/themes/demographie_medicale/statistique_medicale.html

Holdsworth, L. K., \& Webster, V. S. (2004). Direct access to physiotherapy in primary care: now?-and into the future?. Physiotherapy, 90(2), 64-72. doi:10.1016/j.physio.2004.01.005

Holdsworth, L. K., Webster, V. S., \& McFadyen, A. K. (2005). Self-referral to physiotherapy: deprivation and geographical setting - Is there a relationship? Results of a national trial. Physiotherapy, 92(1), 16-25. doi:10.1016/j.physio.2005.11.003

Holdsworth, L. K., Webster, V. S., \& McFadyen, A. K. (2005). Are patients who refer themselves to physiotherapy different from those referred by GPs? Results of a national trial. Physiotherapy, 92(1), 26-33. doi:10.1016/j.physio.2005.11.002 
Huber, O.E., Monnin, D., Paillex, R., Boldi-Goetschy, C., \& Oggier, W. (2011). La Physiothérapie en mutation. Bern: Cahier d'étude de la SSPS.

Jette, D. U., Ardleigh, K., Chandler, K., \& McShea, L. (2006). Decision-Making Ability of Physical Therapists: Physical Therapy Intervention or Medical Referral. Physical Therapy, 86(12), 1619-1629. doi: 10.2522/ptj.20050393

Larequi, Y., Besson, P., Curdy, J., \& Touati, J. (1999). Revue romande de Physiothérapie, accès direct, 16(1).

Le Petit Larousse. (2013). Paris: Larousse.

Leemrisje, C. J., Swinkels, I. C. S., \& Veenhof, C. (2008). Direct access to physical therapy in the Netherlands: Results from the first year in community-based physical therapy. Physical Therapy, 88(8), 936-946. doi:10.2522/ptj.20070308

Logean, S. (2012, 12 janvier). Pénurie de médecins : un chaos programmé. L'HEBDO, pp. 36-41

Ludvigsson, M. L., \& Enthoven, P. (2012). Evaluation of physiotherapists as primary assessors of patients with musculoskeletal disorders seeking primary health care. Physiotherapy, 98(2), 131-137. doi:10.1016/j.physio.2011.04.354

Organisation de Coopération et de Développement Économique. (2013). Panorama de la santé 2011. Accès http://www.oecd-ilibrary.org/sites/health_glance-2011 fr/03/02/index.html;jsessionid=7k1 m175j3bhb2.delta?content Type=\&itemId=/c ontent/chapter/health_glance-2011-21

fr\&containerItemId=/content/serial/19991320\&accessItemIds=/content/book/hea lth_glance-2011-fr\&mimeType=text/html\&site=fr

Pendergast, J., Kliethermes, S., Freburger, J., \& Duffy, P. (2011). A comparison of health care use for physician-referred and self-referred episodes of outpatient physical therapy. Health Services Research, 47(2), 633-654. doi:http://dx.doi.org/10.1111/j.1475-6773.2011.01324.x

Physioswiss. (2011). Rapport annuel2011. Lucerne: Physioswiss.

Physioswiss. (2012). Rapport annuel 2012. Lucerne: Physioswiss. 
Scheermesser, M., Allet, L., Bürge, E., Stegen, C., Nast, I., \& Schämann, A. (2012). Accès direct à la physiothérapie en Suisse : validation linguistique et culturelle d'un questionnaire et position des physiothérapeutes. Kinésithérapie la revue, (124), 29-37.

Seematter-Bagnoud, L., Junod, J., Jaccard Ruedin, H., Roth, M., Foletti, C., \& SantosEggimann, B. (2010). Offre et recours aux soins médicaux ambulatoires en Suisse - Projections à l'horizon 2030 [Document de travail]. Neuchâtel: Observatoire suisse de la santé.

World Confederation for Physical Therapy. (2010). Direct Access. Accès http://www.wcpt.org/node/47869

Moore, J.H., McMillian, D.J., Rosenthal, M.D., \& Weishaar, M.D. (2005). Risk determination for patients with direct access to physical therapy in military health care facilities. Journal of Orthopaedic \& Sports Physical Therapy, 35(10), 674-8. 


\section{Liste des illustrations et tableaux}

Figure 1 : Parcours de soin en Suisse

Figure 2 : Parcours de soin avec AD

Figure 3 : Etudes sélectionnées

Figure 4 : Organigramme de la sélection des articles

Figure 5 : Récapitulatif des outils de mesure présents dans les études

Figure 6 : Tableau de l'outcome « Sexe »

Figure 7 : Tableau de l'outcome « MSQ ou autre »

Figure 8 : Tableau de l'outcome «Diagnostic MSQ »

Figure 9 : Tableau de l'outcome « Durée des symptômes »

Figure 10 : Tableau de l'outcome «Arrêt du TTT »

Figure 11 : Tableau de l'outcome « Réussite des objectifs »

\section{Annexes}

9.1 Structure PICO

$\mathrm{P}=$ Patient

$\mathrm{I}=$ Accès direct

$\mathrm{C}=\quad$ Profil patient $\mathrm{AD}$ vs profil patient MG

$\mathrm{O}=\quad$ Profil type de patient en accès direct

9.2 Equation de recherche

("physical therapy" or physiotherapy) AND ("direct access" or "self-referral" or "first contact") 


\subsection{Echelle de qualité}

\section{Critical Review Form - Quantitative Studies}

CLaw, M., Stewart, D., Pollock, N., Letts, L. Bosch, J., \& Westmorland, M.

McMaster University

Adapted Word Version Used with Permission

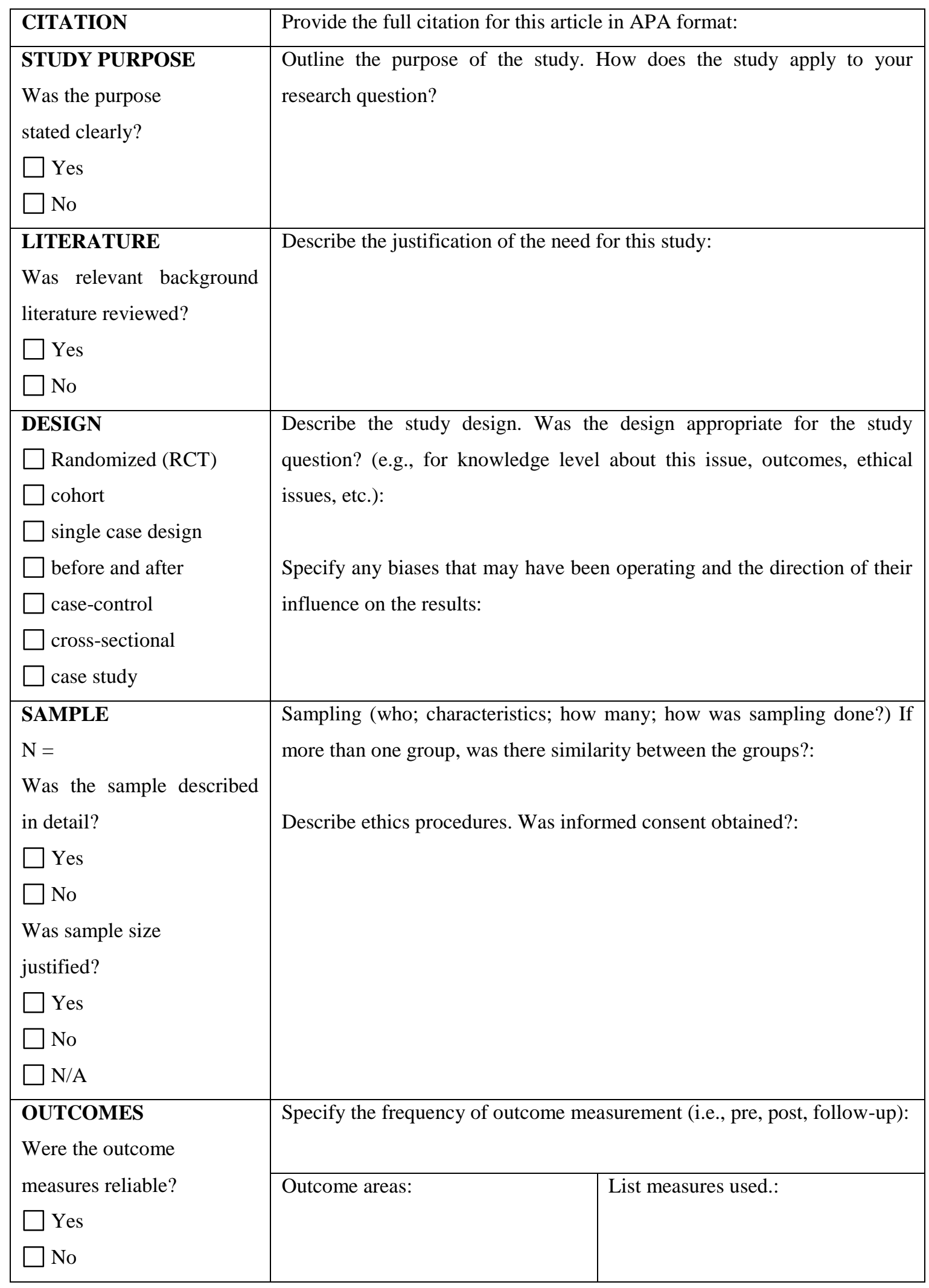




\begin{tabular}{|c|c|}
\hline $\begin{array}{l}\square \text { Not addressed } \\
\text { Were the outcome } \\
\text { measures valid? } \\
\square \text { Yes } \\
\square \text { No } \\
\square \text { Not addressed }\end{array}$ & \\
\hline $\begin{array}{l}\text { INTERVENTION } \\
\text { Intervention was described } \\
\text { in detail? } \\
\square \text { Yes } \\
\square \text { No } \\
\square \text { Not addressed } \\
\text { Contamination } \\
\text { avoided? } \\
\square \text { Yes } \\
\square \text { No } \\
\square \text { Not addressed } \\
\square \text { N/A } \\
\text { Cointervention } \\
\text { avoided? } \\
\square \text { Yes } \\
\square \text { No } \\
\square \text { Not addressed } \\
\square \text { N/A }\end{array}$ & $\begin{array}{l}\text { Provide a short description of the intervention (focus, who delivered it, } \\
\text { how often, setting). Could the intervention be replicated in practice? }\end{array}$ \\
\hline $\begin{array}{l}\text { RESULTS } \\
\text { Results were reported in } \\
\text { terms of statistical } \\
\text { significance? } \\
\square \text { Yes } \\
\square \text { No } \\
\square \text { N/A } \\
\square \text { Not addressed } \\
\text { Were the analysis } \\
\text { method(s) appropriate? } \\
\square \text { Yes } \\
\square \text { No } \\
\square \text { Not addressed }\end{array}$ & $\begin{array}{l}\text { What were the results? Were they statistically significant (i.e., } \mathrm{p}<0.05 \text { )? If } \\
\text { not statistically significant, was study big enough to show an important } \\
\text { difference if it should occur? If there were multiple outcomes, was that } \\
\text { taken into account for the statistical analysis? }\end{array}$ \\
\hline
\end{tabular}




\begin{tabular}{|c|c|}
\hline $\begin{array}{l}\text { Clinical importance was } \\
\text { reported? } \\
\square \text { Yes } \\
\square \text { No } \\
\square \text { Not addressed }\end{array}$ & $\begin{array}{l}\text { What was the clinical importance of the results? Were differences between } \\
\text { groups clinically meaningful? (if applicable) }\end{array}$ \\
\hline $\begin{array}{l}\text { Drop-outs were reported? } \\
\square \text { Yes } \\
\square \text { No }\end{array}$ & $\begin{array}{l}\text { Did any participants drop out from the study? Why? (Were reasons given } \\
\text { and were drop-outs handled appropriately?) }\end{array}$ \\
\hline $\begin{array}{ll}\text { CONCLUSIONS } & \text { AND } \\
\text { IMPLICATIONS } & \\
\text { Conclusions } & \text { were } \\
\text { appropriate given } & \text { study } \\
\text { methods and results } & \\
\square \text { Yes } & \\
\square \text { No } & \end{array}$ & $\begin{array}{l}\text { What did the study conclude? What are the implications of these results for } \\
\text { practice? What were the main limitations or biases in the study? }\end{array}$ \\
\hline
\end{tabular}




\subsection{Echelle de qualité modifiée}

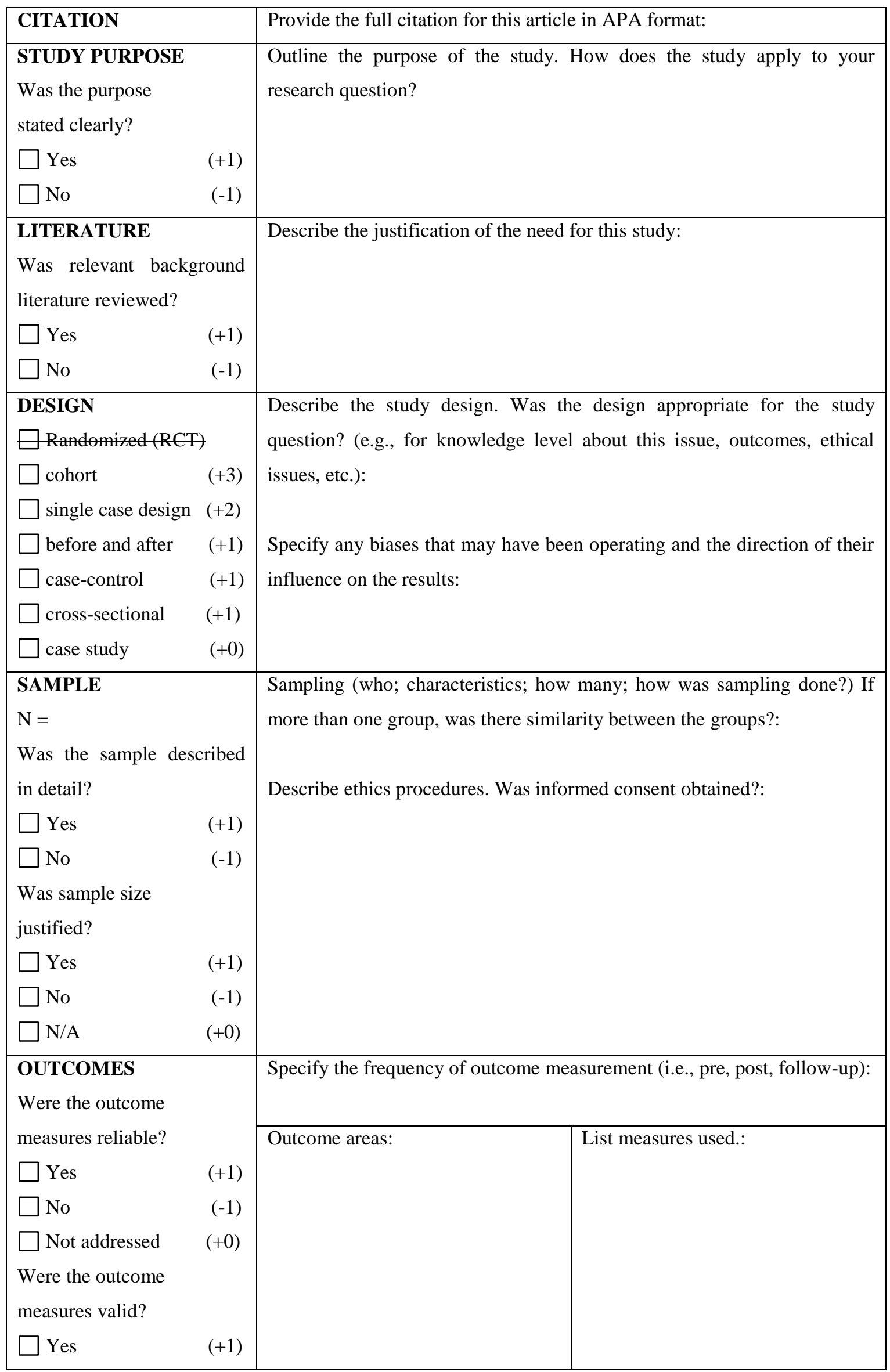




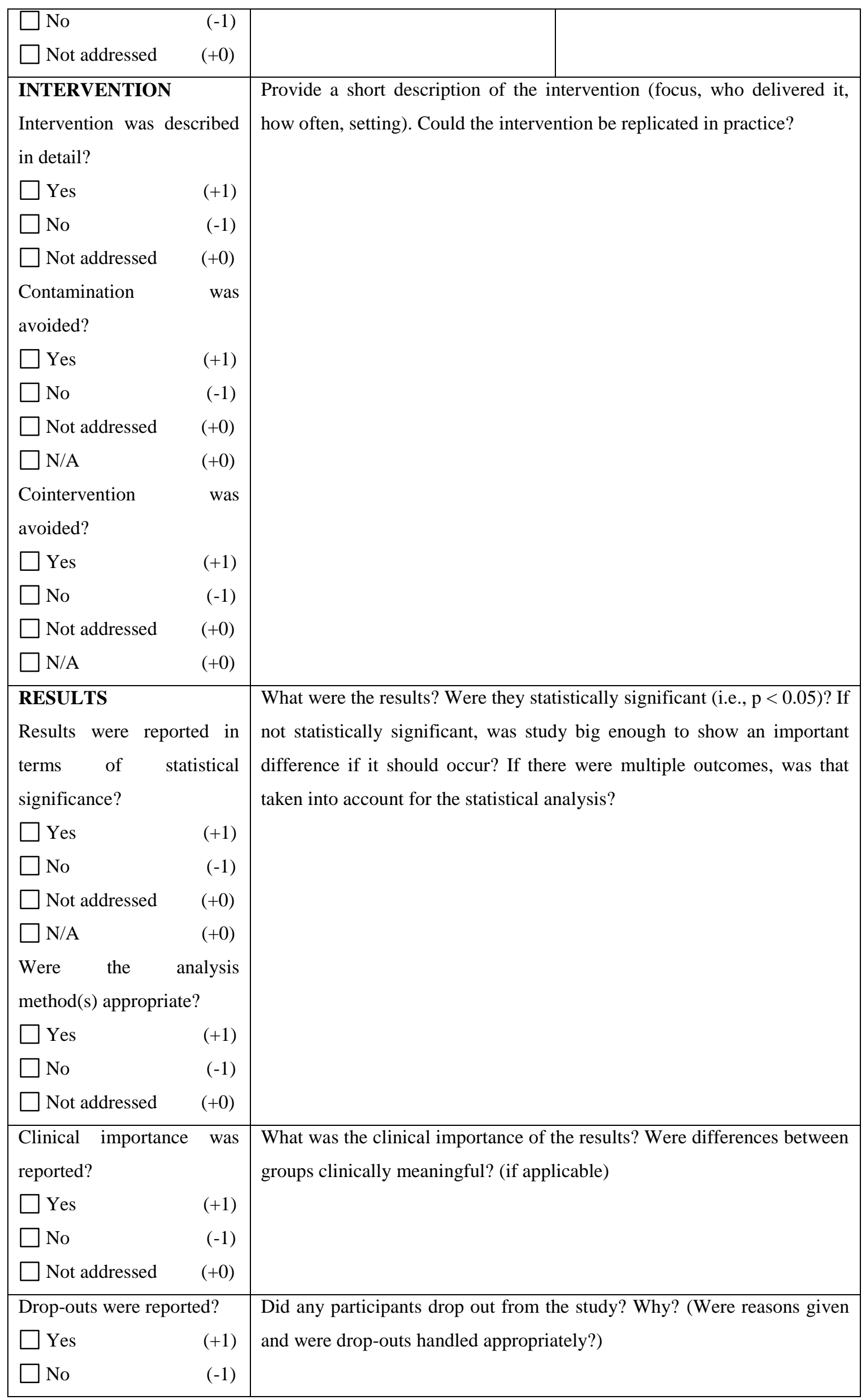




\begin{tabular}{|lr|l|}
\hline CONCLUSIONS & AND & What did the study conclude? What are the implications of these results for \\
IMPLICATIONS & & practice? What were the main limitations or biases in the study? \\
$\begin{array}{l}\text { Conclusions } \\
\text { appropriate given }\end{array}$ & study & \\
methods and results & & \\
$\square$ Yes & $(+1)$ & \\
$\square$ No & $(-1)$ & \\
\hline
\end{tabular}


9.5 Grille d'extraction des données

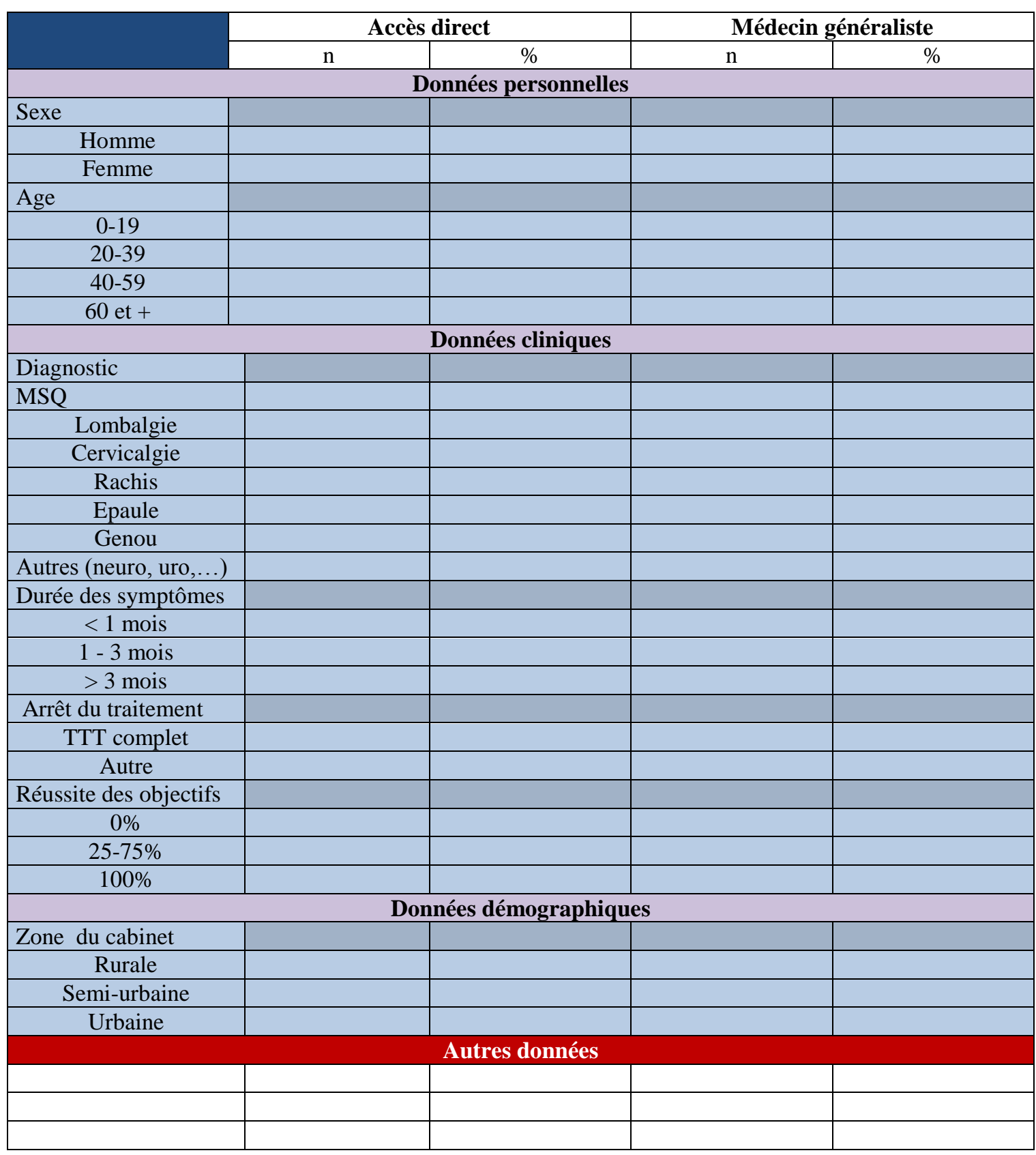


9.6 Résultats comparables aux cinq études

\begin{tabular}{|c|c|c|c|c|}
\hline \multirow{4}{*}{$\begin{array}{l}\text { Echantillon } 5 \\
\text { études }\end{array}$} & Accès direct & 21198 & & \\
\hline & $\begin{array}{c}\text { Médecin } \\
\text { généraliste }\end{array}$ & 54802 & & \\
\hline & \multicolumn{2}{|c|}{ Accès direct } & \multicolumn{2}{|c|}{ Médecin généraliste } \\
\hline & $\mathrm{n}$ & $\%$ & $\bar{n}$ & $\%$ \\
\hline \multicolumn{5}{|c|}{ Données personnelles } \\
\hline \multicolumn{5}{|l|}{ Sexe } \\
\hline Homme & 8768 & 41.4 & 22025 & 40.2 \\
\hline Femme & 12430 & 58.6 & 32777 & 59.8 \\
\hline \multicolumn{5}{|l|}{ Age } \\
\hline \multicolumn{5}{|c|}{ Données cliniques } \\
\hline \multicolumn{5}{|l|}{ Diagnostic } \\
\hline MSQ & 17675 & 84.4 & 44236 & 81.2 \\
\hline $\begin{array}{c}\text { Autres (neuro, } \\
\text { uro }, . . .)\end{array}$ & 3275 & 15.6 & 10221 & 18.8 \\
\hline
\end{tabular}

9.7 Résultats comparables à quatre études

\begin{tabular}{|c|c|c|c|c|}
\hline \multirow{4}{*}{ Echantillon 4 études } & Accès direct & 3701 & & \\
\hline & $\begin{array}{l}\text { Médecin } \\
\text { généraliste }\end{array}$ & 9592 & & \\
\hline & \multicolumn{2}{|c|}{ Accès direct } & \multicolumn{2}{|c|}{ Médecin généraliste } \\
\hline & $\mathrm{n}$ & $\%$ & $\mathrm{n}$ & $\%$ \\
\hline \multicolumn{5}{|c|}{ Données cliniques } \\
\hline \multicolumn{5}{|l|}{ Diagnostic } \\
\hline Membre supérieur & 276 & 12.5 & 872 & 18.5 \\
\hline Epaule & 228 & 82.6 & 698 & 80.0 \\
\hline Rachis & 1733 & 78.5 & 3327 & 70.5 \\
\hline Lombalgie & 733 & 42.3 & 1360 & 40.9 \\
\hline Cervicalgie & 682 & 39.4 & 1543 & 46.4 \\
\hline Membre inférieur & 199 & 9.0 & 523 & 11.1 \\
\hline Genou & 92 & 46.2 & 256 & 48.9 \\
\hline \multicolumn{5}{|l|}{ Durée des symptômes } \\
\hline$<1$ mois & 1733 & 47.4 & 3167 & 33.6 \\
\hline $1-3$ mois & 748 & 20.5 & 2209 & 23.5 \\
\hline$>3$ mois & 1175 & 32.1 & 4042 & 42.9 \\
\hline \multicolumn{5}{|l|}{ Arrêt du traitement } \\
\hline TTT complet & 1759 & 83.6 & 4477 & 75.4 \\
\hline $\begin{array}{l}\text { TTT incomplet ou } \\
\text { arrêté }\end{array}$ & 189 & 9.0 & 809 & 13.6 \\
\hline Autre & 156 & 7.4 & 651 & 11.0 \\
\hline \multicolumn{5}{|l|}{ Réussite des objectifs } \\
\hline $0 \%$ & 52 & 3.1 & 301 & 6.5 \\
\hline $25-75 \%$ & 362 & 21.9 & 1222 & 26.4 \\
\hline $100 \%$ & 1237 & 74.9 & 3107 & 67.1 \\
\hline
\end{tabular}




\section{Physiothérapie suisse : vers un accès direct?}

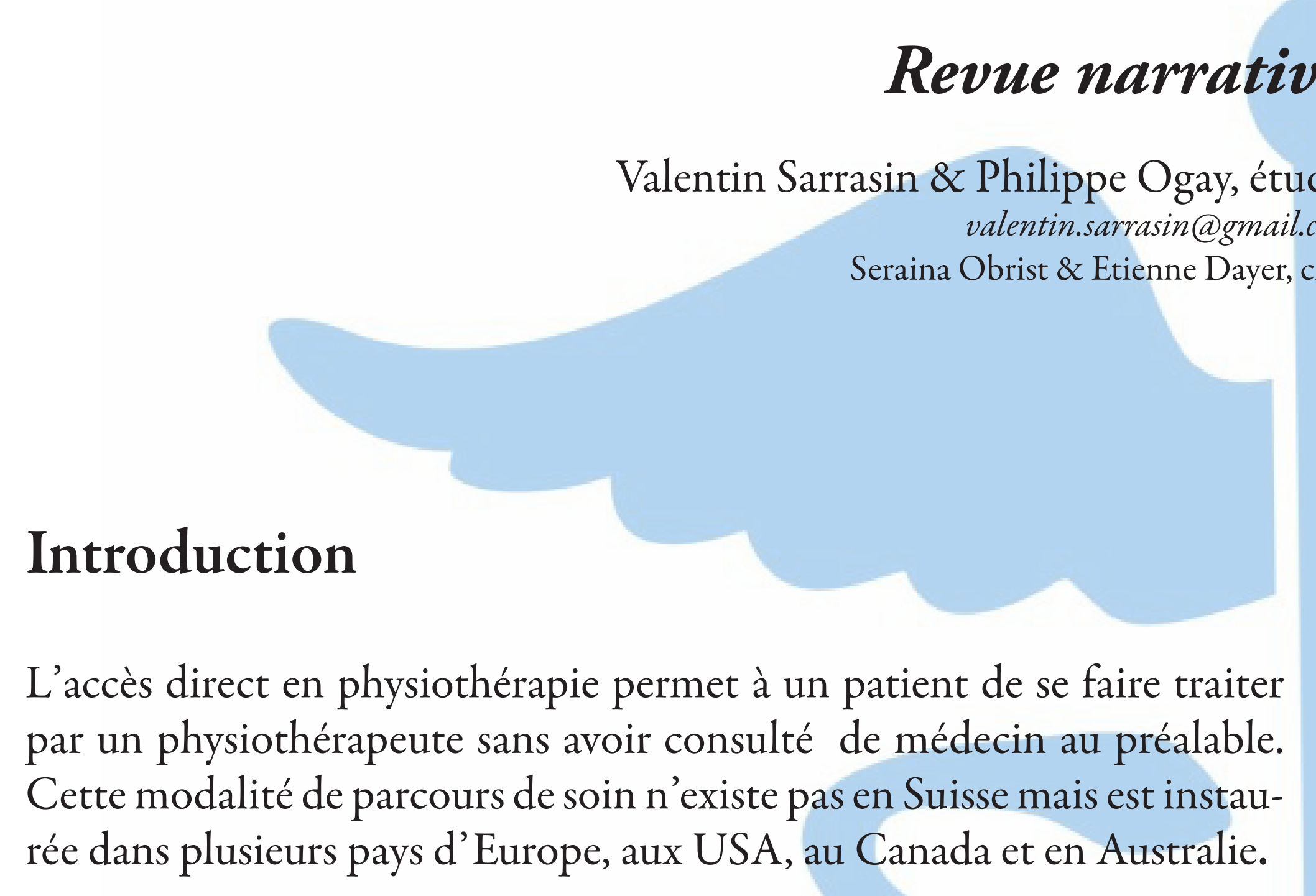

\section{Objectifs}

Définir s'il existe une différence entre le profil d'une personne consultant son physiothérapeute en accès direct et celui d'un patient qui sollicite son médecin au préalable. Puis, selon nos résultats, définir un profil type de patient consultant son physiothérapeute en accès direct.

\section{Méthode}

Les moteurs de recherche Pubmed, Web of Knowledge, Science Direct, Psychinfo, Cochrane, PEDro, Medline, Francis et CINAHL ont été consultés, donnant un total de 482 articles. Après une sélection comportant trois tris et une évaluation de la qualité avec une version modifiée de l'échelle «Critical Review Form» de Law, cinq articles ont été retenus.

\section{Résultats}

Les résultats montrent une différence de profil entre les deux groupes, caractérisée par les critères :

- Âge : la clientèle est plus jeune en accès direct

- Diagnostic : les physiothérapeutes traitent surtout les troubles du rachis

- Durée des symptômes : la durée est plus courte en accès direct

- Arrêt du traitement : il y a un taux plus élevés de traitements ache-

vés en accès direct

Réussite des objectifs : pour une réussite à 100\% des objectifs, le taux est plus élevé en accès direct

\section{Résultats comparables aux cinq études}

\begin{tabular}{|c|c|c|c|c|}
\hline \multirow{4}{*}{$\begin{array}{c}\text { Echantillon } 5 \\
\text { études }\end{array}$} & Accès direct & 21198 & & \\
\hline & $\begin{array}{l}\text { Médecin } \\
\text { généraliste }\end{array}$ & 54802 & & \\
\hline & \multicolumn{2}{|c|}{ Accès direct } & \multicolumn{2}{|c|}{ Médecin généraliste } \\
\hline & $\mathrm{n}$ & $\%$ & $\mathrm{n}$ & $\%$ \\
\hline \multicolumn{5}{|c|}{ Données personnelles } \\
\hline \multicolumn{5}{|c|}{ Sexe } \\
\hline Hommes & 8768 & 41.4 & 22025 & 40.2 \\
\hline Femmes & 12430 & 58.6 & 32777 & 59.8 \\
\hline \multicolumn{5}{|c|}{ Données cliniques } \\
\hline \multicolumn{5}{|l|}{ Diagnostic } \\
\hline MSQ & 17675 & 84.4 & 44236 & 81.2 \\
\hline $\begin{array}{c}\text { Autres (neuro, } \\
\text { uro,...) }\end{array}$ & 8768 & 41.4 & 22025 & 40.2 \\
\hline
\end{tabular}

Résultats comparables à quatre études

\begin{tabular}{|c|c|c|c|c|}
\hline \multirow[t]{3}{*}{$\begin{array}{l}\text { Echantillon } 4 \\
\text { études }\end{array}$} & $\begin{array}{l}\text { Accès direct } \\
\text { Médecin } \\
\text { généraliste }\end{array}$ & $\begin{array}{l}3701 \\
9592 \\
\end{array}$ & & \\
\hline & \multicolumn{2}{|c|}{ Accès direct } & \multicolumn{2}{|c|}{ Médecin généraliste } \\
\hline & $\mathrm{n}$ & $\%$ & $\mathrm{n}$ & $\%$ \\
\hline \multicolumn{5}{|c|}{ Données cliniques } \\
\hline \multicolumn{5}{|l|}{ Diagnostic } \\
\hline Membre supérieur & 276 & 12.5 & 872 & 18.5 \\
\hline Epaule & 228 & 82.6 & 698 & 80.0 \\
\hline Rachis & 1733 & 78.5 & 3327 & 70.5 \\
\hline Lombalgie & 733 & 42.3 & 1360 & 40.9 \\
\hline Cervicalgie & 682 & 39.4 & 1543 & 46.4 \\
\hline Membre inférieur & 199 & 9.0 & 523 & 11.1 \\
\hline Genou & 92 & 46.2 & 256 & 48.9 \\
\hline \multicolumn{5}{|c|}{ Durée des symptômes } \\
\hline$<1$ mois & 1733 & 47.4 & 3167 & 33.6 \\
\hline $1-3$ mois & 748 & 20.5 & 2209 & 23.5 \\
\hline$>3$ mois & 1175 & 32.1 & 4042 & 42.9 \\
\hline \multicolumn{5}{|c|}{ Arrêt du traitement } \\
\hline TTT complet & 1759 & 83.6 & 4477 & 75.4 \\
\hline $\begin{array}{l}\text { TTT incomplet } \\
\text { ou arrêté }\end{array}$ & 189 & 9.0 & 809 & 13.6 \\
\hline Autre & 156 & 7.4 & 651 & 11.0 \\
\hline \multicolumn{5}{|c|}{ Réussite des objectifs } \\
\hline $0 \%$ & 52 & 3.1 & 301 & 6.5 \\
\hline $25 \%-75 \%$ & 362 & 21.9 & 1222 & 26.4 \\
\hline $100 \%$ & 1237 & 74.9 & 3107 & 67.1 \\
\hline
\end{tabular}

\section{Conclusion}

Le profil type d'une personne consultant son physiothérapeute en accès direct se démarque par une donnée personnelle - l'âge - et une donnée clinique - le diagnostic. L'accès direct offre de nombreux avantages tels que des soins rapides et de qualité, une diminution des coûts de la santé et une amélioration de la collaboration entre les physiothérapeutes et les autres professionnels de la santé.

\section{Bibliographie}

